\title{
Synthesis and biological evaluation of new 1,3-thiazolidine-4-one derivatives of nitro-L-arginine methyl ester
}

\author{
Andreea-Teodora Pânzariu1, Maria Apotrosoaei ', Ioana Mirela Vasincu', Maria Drăgan', Sandra Constantin 1, \\ Frédéric Buron ${ }^{2}$, Sylvain Routier ${ }^{2}$, Lenuta Profire ${ }^{1 *}$ and Cristina Tuchilus ${ }^{3}$
}

\begin{abstract}
Background: L-Arginine is a semi-essential aminoacid with important role in regulation of physiological processes in humans. It serves as precursor for the synthesis of proteins and is also substrate for different enzymes such as nitric oxide synthase. This amino-acid act as free radical scavenger, inhibits the activity of pro-oxidant enzymes and thus acts as an antioxidant and has also bactericidal effect against a broad spectrum of bacteria.

Results: New thiazolidine-4-one derivatives of nitro-L-arginine methyl ester $\left(\mathrm{NO}_{2}\right.$-Arg-OMe) have been synthesized and biologically evaluated in terms of antioxidant and antibacterial/antifungal activity. The structures of the synthesized compounds were confirmed by ${ }^{1} \mathrm{H},{ }^{13} \mathrm{C}$ NMR, Mass and IR spectral data. The antioxidant potential was investigated using in vitro methods based on ferric/phosphomolybdenum reducing antioxidant power and DPPH/ ABTS radical scavenging assay. The antibacterial effect was investigated against Gram positive (Staphylococcus aureus ATCC 25923, Sarcina lutea ATCC 9341) and Gram negative (Escherichia coli ATCC 25922, Pseudomonas aeruginosa ATCC 27853) bacterial strains. The antifungal activity was also investigated against Candida spp. (Candida albicans ATCC 10231, Candida glabrata ATCC MYA 2950, Candida parapsilosis ATCC 22019).
\end{abstract}

Conclusions: Synthesized compounds showed a good antioxidant activity in comparison with the $\mathrm{NO}_{2}$-Arg-OMe. The antimicrobial results support the selectivity of tested compounds especially on $P$. aeruginosa as bacterial strain and C. parapsilosis as fungal strain. The most proper compounds were $\mathbf{6 g}\left(\mathrm{R}=3-\mathrm{OCH}_{3}\right)$ and $\mathbf{6} \mathbf{h}\left(\mathrm{R}=2-\mathrm{OCH}_{3}\right)$ which showed a high free radical (DPPH, ABTS) scavenging ability and $\mathbf{6 j}\left(\mathrm{R}=2-\mathrm{NO}_{2}\right)$ that was the most active on both bacterial and fungal strains and also it showed the highest ABTS radical scavenging ability.

Keywords: Nitro-L-arginine methyl ester, 1,3-Thiazolidine-4-one, Spectral methods, Antioxidant effects, Antibacterial/antifungal activity

\section{Background}

L-Arginine is an amino acid with the highest nitrogen content known for its important role in regulation of physiological processes in humans [1]. This amino acid is considered a semi-essential amino acid because normal cells can not only synthesize arginine de novo through the ornithine cycle but also uptake extracellular arginine

\footnotetext{
*Correspondence: lenuta.profire@umfiasi.ro

1 Department of Pharmaceutical Chemistry, Faculty of Pharmacy, University of Medicine and Pharmacy "Grigore T. Popa", 16 University Street, 700115 lasi, Romania

Full list of author information is available at the end of the article
}

[2]. It serves as a precursor for the synthesis of proteins and it is also substrate for different enzymes. For example nitric oxide synthase (NOS) converts arginine to nitric oxide (NO) and citrulline. Three isoforms of NOS have been described: endothelial NOS (eNOS), neuronal NOS (nNOS), that are constitutive isoforms (cNOS) and inducible NOS (iNOS) [3]. NO, is an important signal molecule, involved in immune responses, angiogenesis, epithelialization and formation of granulation tissue, vasodilatation of smooth muscle and inhibition of platelets activation/aggregation $[4,5]$. The cNOS produce NO in picomolar amounts for short time, being responsible

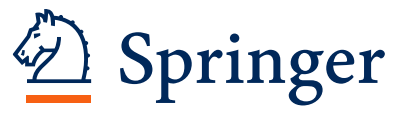

(c) 2016 Pânzariu et al. This article is distributed under the terms of the Creative Commons Attribution 4.0 International License (http://creativecommons.org/licenses/by/4.0/), which permits unrestricted use, distribution, and reproduction in any medium, provided you give appropriate credit to the original author(s) and the source, provide a link to the Creative Commons license, and indicate if changes were made. The Creative Commons Public Domain Dedication waiver (http://creativecommons.org/ publicdomain/zero/1.0/) applies to the data made available in this article, unless otherwise stated. 
for regulation of arterial blood pressure, while iNOS produces large amounts of NO through cell activation under inflammatory conditions, appearing to be involved in pathophysiological phenomena [3]. Nitro-L-arginine methyl ester $\left(\mathrm{NO}_{2}\right.$-Arg-OMe, L-NAME) is known as selective inhibitor of inducible NOS, which showed antinociceptive effects in mice and reversed thermal hyperalgesia in rats with carrageenan arthritis [6]. It was also reported that L-NAME attenuates the withdrawal from cocaine [7] and prevents the behaviour effects indused by phencyclidin, a dissociative drug [8].

L-Arginine is reported also to act as free radical scavenger, inhibits the activity of pro-oxidant enzymes and thus acts as an antioxidant $[9,10]$. This endogenous molecule has also bactericidal effect against a broad spectrum of bacteria, by nitrosation of cysteine and tyrosine residues, which lead to dysfunction of bacterial proteins. This effect could be useful in different conditions as wounds when infection could delay the healing process. The two most common bacteria in wounds are Pseudomonas aeruginosa and Staphylococcus aureus [11]. In addition, to its role as precursor of $\mathrm{NO}, \mathrm{L}$-arginine can be metabolized by arginase to ornithine and urea. Ornithine is an essential precursor for collagen and polyamines synthesis, both required for wound healing processes [12]. Based on all these aspects there has been reported that L-arginine has important roles in Alzheimer disease [13], inflammatory process [14], healing and tissue regeneration [14-16] and also it showed anti-atherosclerotic activity $[17,18]$.

On other hand the heterocyclic compounds are an integral part in organic chemistry field and constitute a modern research field that is being currently pursued by many research teams [19]. Diversity in the biological response of 1,3-thiazolidine-4-one derivatives had attracted the attention of many researchers for a thorough exploration of their biological potential. These compounds have been reported for their antioxidant [20-22], anti-inflammatory [23], antibacterial/antifungal [24-26], antitumor [27], antidiabetic [28], antihyperlipidemic [29] and antiarthritic [30] effects.

In order to improve the biological effects of L-arginine and, new 1,3-thiazolidine-4-one derivatives have been synthesized. The spectral data (FT-IR, ${ }^{1} \mathrm{H}-\mathrm{NMR}$,
${ }^{13} \mathrm{C}$-NMR, MS) of each compound were recorded and the compounds were screened for their in vitro antioxidant potential and antibacterial/antifungal activity.

\section{Results and discussion}

\section{Chemistry}

The synthesis of thiazolidine-4-one compounds derived from $\mathrm{L}-\mathrm{NO}_{2}$-Arg-OMe was performed in two steps and is summarized in Scheme 1 and Table 1. The first step consisted in formation of the 1,3-thiazolidin-4-one cycle via a one-pot condensation/cyclization reaction which implies the using of ethyl 3-aminopropionate hydrochloride $\mathbf{1}$, different substituted aromatic aldehydes $\mathbf{2 a}-\mathbf{j}$ and thioglycolic acid 3 using a similar approach described in our previous work [27]. The product of this reaction was treated with $\mathrm{KOH}$ to give compounds $\mathbf{4}$ in satisfactory to very good overall yields. In the second and last step, the formation of amide bond between acid derivatives 4 and $N_{\omega}$ nitro-L-arginine methyl ester hydrochloride 5 was carried out using classical conditions in presence of 1-ethyl-3-(3dimethylaminopropyl)carbodiimide hydrochloride (EDC) and 1-hydroxybenzotriazole (HOBt) to lead to new thiazolidine-4-one derivatives with arginine moiety $\mathbf{6 a}-\mathbf{j}$.

The structure of the compounds was assigned on the basis of spectral data (IR, ${ }^{1} \mathrm{H}-\mathrm{NMR},{ }^{13} \mathrm{C}-\mathrm{NMR}, \mathrm{MS}$ ) which are provided in the Experimental Section. The spectral data for compounds $\mathbf{4 a - j}$ were presented in our previous paper [31].

The analysis of IR spectral data obtained for compounds $6 \mathbf{a}-\mathbf{j}$ showed that the NH group corresponding to the amide bond formed was identified between 3305 and $3294 \mathrm{~cm}^{-1}$ in the form of a medium or low intensity bands. The specific anti-symmetric valence vibration of $\mathrm{CH}_{2}$ group has been reported in the range of 2940$2825 \mathrm{~cm}^{-1}$ and overlaps with specific absorption band of $\mathrm{CH}$ group, which is identified in the same range. The $\mathrm{C}=\mathrm{O}$ group was identified as three absorption bands: the absorption band in the $1760-1670 \mathrm{~cm}^{-1}$ corresponds to ester group $\left(\mathrm{COOCH}_{3}\right)$, in the area of $1686-1647 \mathrm{~cm}^{-1}$ was identified the absorption band corresponding to $\mathrm{C}=\mathrm{O}$ from amide bond and the group $\mathrm{C}=\mathrm{O}$ from the thiazolidine-4-one moiety appears in the range of

$$
\text { (a) }
$$

Scheme 1 Synthesis of compounds $\mathbf{6 a}-\mathbf{j}$. Reagents and conditions: a DIPEA, toluene, reflux 24-30 h; b KOH 1 M, EtOH/THF (1/1), r.t. 8-12 h then $\mathrm{HCl} 1 \mathrm{M} ; \mathbf{c} \mathrm{N}_{\omega}$-nitro-L-arginine methyl ester hydrochloride (5), HOBt, EDC, DCM, r.t. 10-15 h 
Table 1 Synthesis of derivatives 4 and 6

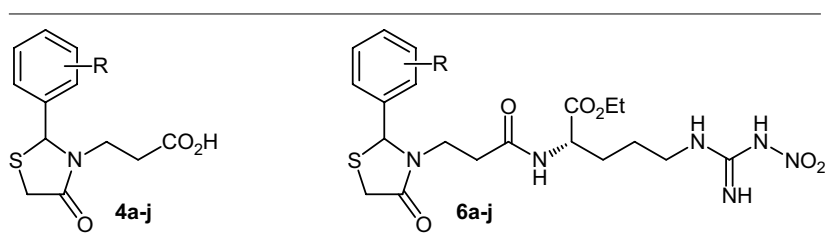

\begin{tabular}{|c|c|c|c|c|}
\hline Entry & Comp. & $\mathbf{R}$ & 4, Yield ${ }^{\mathrm{a}}(\%)$ & 6, Yield $^{\mathrm{a}}(\%)$ \\
\hline 1 & $\mathbf{a}$ & $\mathrm{H}$ & 73 & 93 \\
\hline 2 & b & $4-\mathrm{CH}_{3}$ & 55 & 91 \\
\hline 3 & c & $4-\mathrm{Cl}$ & 59 & 89 \\
\hline 4 & d & $4-F$ & 67 & 75 \\
\hline 5 & e & $4-\mathrm{Br}$ & 78 & 87 \\
\hline 6 & f & $4-\mathrm{OCH}_{3}$ & 55 & 86 \\
\hline 7 & $\mathbf{g}$ & $3-\mathrm{OCH}_{3}$ & 57 & 78 \\
\hline 8 & $\mathbf{h}$ & $2-\mathrm{OCH}_{3}$ & 64 & 76 \\
\hline 9 & $\mathbf{i}$ & $3-\mathrm{NO}_{2}$ & 63 & 50 \\
\hline 10 & $\mathbf{j}$ & $2-\mathrm{NO}_{2}$ & 82 & 91 \\
\hline
\end{tabular}

a Yields are indicated in isolated compounds

$1647-1610 \mathrm{~cm}^{-1}$. The vibration of $\mathrm{C}-\mathrm{S}$ bond, specific for thiazolidine-4-one, was identified between 694 and $668 \mathrm{~cm}^{-1}$.

The formation of $\mathbf{6 a}-\mathbf{j}$ has also been proved by the NMR data. The thiazolidine-4-one structure was proved by characteristic proton signals. The proton of $\mathrm{S}-\mathrm{CH}-\mathrm{N}$ group appears as doublet in the range of 5.72-6.08 while the two protons from thio-methylene group $\left(\mathrm{S}-\mathrm{CH}_{2}\right)$ were recorded dispersed; the first resonates between 4.41 and $4.72 \mathrm{ppm}$, and the second between 3.80 and $4.07 \mathrm{ppm}$. The amide bond $(-\mathrm{NH}-\mathrm{CO})$ was proved by the characteristic proton signal which resonates as singlet in the range $8.48-8.68 \mathrm{ppm}$.

In the ${ }^{13} \mathrm{C}-\mathrm{NMR}$ spectra the carbons of thiazolidine4-one system appear between 64.36 and $62.65 \mathrm{ppm}$ for $\mathrm{S}-\underline{\mathrm{C}} \mathrm{H}-\mathrm{N}$ and between 34.53 and $33.10 \mathrm{ppm}$ for $-\underline{\mathrm{CH}}_{2}-\mathrm{S}$. The signals for the three $\mathrm{CO}$ groups $\left(\mathrm{CO}_{\text {thiazolidine, }} \mathrm{CO}_{\mathrm{am}-}\right.$ ide, $\mathrm{CO}_{\text {ester }}$ ) appear in the range of $173.24-160.39 \mathrm{ppm}$, which confirm the success of peptide coupling reaction.

The proton and carbon signals for other characteristics groups were observed according to the expected chemical shift and integral values. The NMR spectral data coupled with mass spectra strong support the proposed structures of each synthesized compounds.

\section{Biological evaluation}

\section{Antioxidant activity}

The antioxidant activity was evaluated using in vitro tests: DPPH and ABTS radical scavenging, phosphomolydenum reducing antioxidant power and ferric reducing antioxidant power assays. For each compound it was calculated effective concentration $50\left(\mathrm{EC}_{50}\right)$ by linear regression. The results were expressed as $\mathrm{EC}_{50}$ value which represents the concentration where half of the substrate is being reduced by the tested compounds.

\section{The DPPH radical scavenging assay}

The purple free radical DPPH (2,2-diphenyl-1-(2,4,6trinitrophenyl)hydrazyl) is a stable compound that can be scavenged through antioxidants by reduction to 2,2-diphenyl-1-(2,4,6-trinitrophenyl)hydrazine), a colorless or yellow product visible at $517 \mathrm{~nm}$ [32]. The scavenging activities (\%) of thiazolidine-4-one derivatives of nitro-L-arginine methyl ester $\mathbf{6} \mathbf{a}-\mathbf{j}$ at different concentrations $(0.33,0.66,0.99$ and $1.32 \mathrm{mg} / \mathrm{mL})$ are presented in Fig. 1 . The high values of the scavenging activity indicate a good antiradical effect. The results expressed as $\mathrm{EC}_{50}$ values $(\mathrm{mg} / \mathrm{mL})$ are shown in Table 2 . Low values of $\mathrm{EC}_{50}$ demonstrate a higher scavenging ability.

It was observed that 1,3-thiazolidine-4-one derivatives of methyl ester of nitro-L-arginine $\left(\mathrm{NO}_{2}\right.$-Arg-OMe) showed an improved scavenging ability compared to parent molecule $\left(\mathrm{NO}_{2}\right.$-Arg-OMe) and L-arginine, excepting nitro substituted derivatives $\mathbf{6 i}$ and $\mathbf{6 j}$, which showed comparable antiradical activity. It is also noted that the antiradical activity increases with the concentration, the highest inhibition being recorded at the concentration of $1.32 \mathrm{mg} / \mathrm{mL}$. At this concentration the inhibition rate ranged from $22.62 \%$ for $\mathbf{6 d}(\mathrm{R}=4-\mathrm{F})$ up to $42.61 \%$ for 6h $\left(\mathrm{R}=2-\mathrm{OCH}_{3}\right)$ and $47.63 \%$ for $\mathbf{6} \mathbf{a}(\mathrm{R}=\mathrm{H})$.

The scavenging ability depends on the substituent of phenyl ring of thiazolidine-4-one moiety. The most active compound was unsubstituted derivative $\mathbf{6 a}$ $\left(\mathrm{EC}_{50}=1.7294 \pm 0.048\right)$, which is 1.6 times more active than $\mathrm{NO}_{2}$-Arg-OMe $\left(\mathrm{EC}_{50}=2.7163 \pm 0.019\right)$. A good influence was showed also by the methoxy substitution in ortho and meta position, the corresponding compounds 6h $\left(2-\mathrm{OCH}_{3}, \mathrm{EC}_{50}=1.8068 \pm 0.028\right)$ and $\mathbf{6 g}\left(3-\mathrm{OCH}_{3}\right.$, $\left.\mathrm{EC}_{50}=1.8868 \pm 0.013\right)$ being 1.5 times more active than $\mathrm{NO}_{2}$-Arg-OMe. All tested compounds were less active than vitamin $\mathrm{E}$ used as a positive control.

\section{The ABTS radical scavenging assay}

The radical of 2,2'-azinobis-(3-ethylbenzothiazoline6-sulfonic acid) $\left(\mathrm{ABTS}^{+}\right)$generated by oxidation of ABTS with potassium persulfate is reduced in the presence of hydrogen-donating compounds. The influence of concentration of the antioxidant and duration of reaction on the radical cation absorption inhibition are taken into account for antioxidant activity evaluation [33]. The antioxidants produce a discoloration with a decrease in the absorbance measured at $734 \mathrm{~nm}$ [34]. 


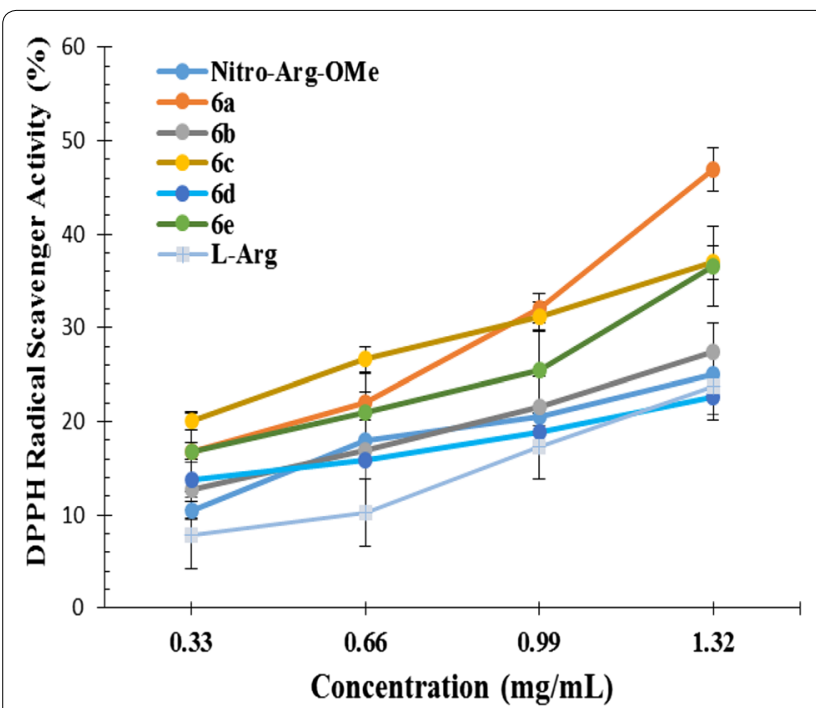

Fig. 1 The DPPH radical scavenging ability (\%) of derivatives $\mathbf{6} \mathbf{a}-\mathbf{j}$

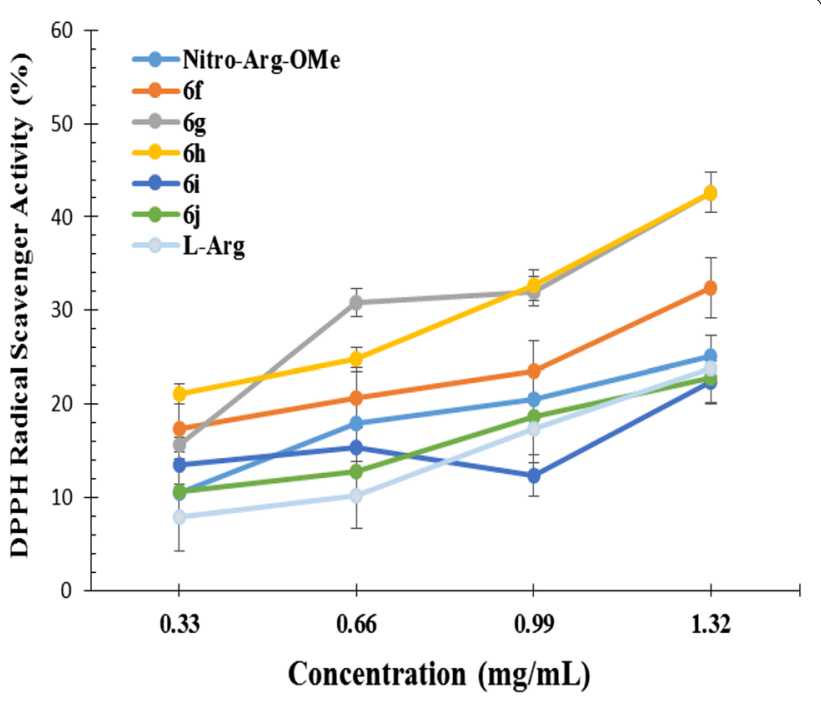

The activity is depending on the substitution of phenyl ring of thiazolidine-4-one scaffold (Table 3). The most active compounds were $\mathbf{6 j}, \mathbf{6 g}$ and $\mathbf{6 h}$ that have nitro in ortho position and methoxy in ortho and para position respectively. These compounds are 35 times $\left(\mathbf{6 j}, \mathrm{EC}_{50}=0.0525 \pm 0.015\right), 22$ times $\left(\mathbf{6 g}, \mathrm{EC}_{50}=0.0827 \pm 0.017\right)$ and 20 times $(\mathbf{6 h}$, $\left.\mathrm{EC}_{50}=0.0918 \pm 0.032\right)$ more active than $\mathrm{NO}_{2}$-ArgOMe $\left(\mathrm{EC}_{50}=1.8487 \pm 0.026\right)$. A very good activity was showed also by the compounds $\mathbf{6 c}$ and $\mathbf{6 d}$ that have chloro and fluoro in para postion of phenyl ring. They are 10 times $\left(\mathbf{6 c}, \mathrm{EC}_{50}=0.1885 \pm 0.014\right)$ and 11 times $(\mathbf{6 d}$, $\left.\mathrm{EC}_{50}=0.1720 \pm 0.018\right)$ respectively more active than $\mathrm{NO}_{2}$-Arg-OMe. It is also noted that all tested compounds are more active than $\mathrm{L}$-arginine but less active than vitamin $\mathrm{E}$ used as a positive control.

\section{Phosphomolydenum reducing antioxidant power (PRAP) assay}

The total antioxidant activity was determined by the formation of phosphomolybdenum blue complex by the reduction of $\mathrm{Mo}^{6+}$ to $\mathrm{Mo}^{5+}$ under the action of electron donating compounds. The maximum absorption of the complex was recorded at $695 \mathrm{~nm}$ and the reducing antioxidant effectiveness is correlated with high absorbance values [35]. The graphical representation of the absorbance values at different concentrations $(0.18,0.36,0.54$ and $0.72 \mathrm{mg} / \mathrm{mL})$ is shown in Fig. 3. As we expected, the absorbance of $\mathbf{6 a}-\mathbf{j}$ increases with the concentration, the highest absorbance/ activity being recorded at the concentration of $0.72 \mathrm{mg} / \mathrm{mL}$.

The data support the positive influence of thiazolidine-4-one moiety for increase the antioxidant effect than the DPPH inhibition percentage. 


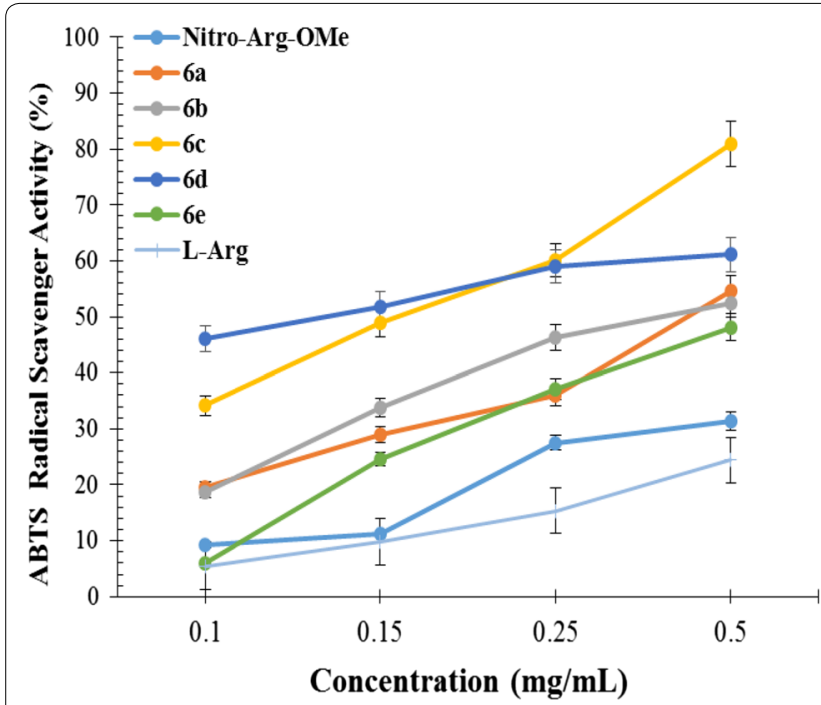

Fig. 2 The ABTS radical scavenging ability (\%) of derivatives $\mathbf{6 a - j}$

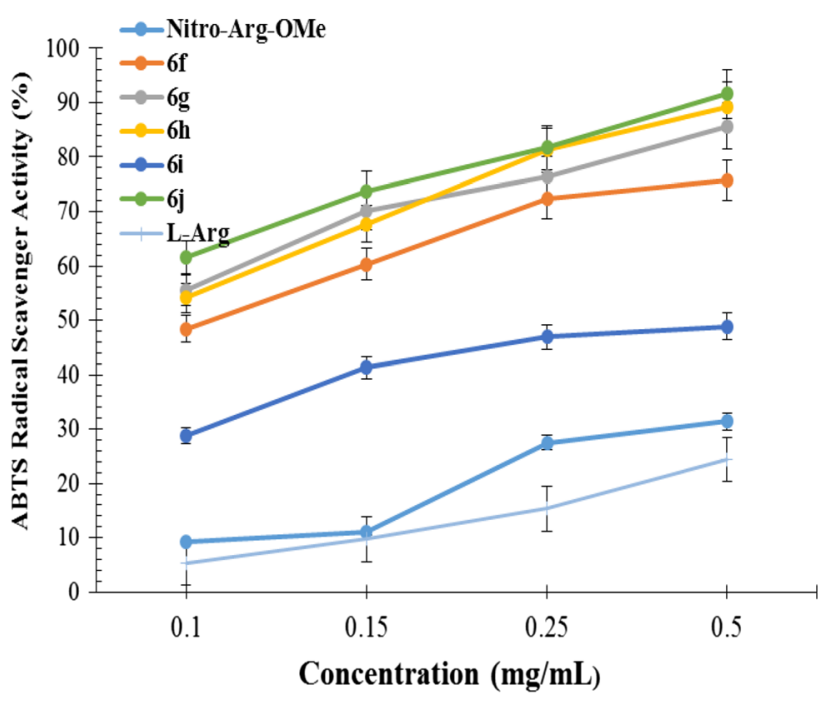

Table 3 The ABTS scavenging ability $\left(E C_{50} \mathrm{mg} / \mathrm{mL}\right.$ ) of derivatives $6 \mathbf{a}-\mathbf{j}$

\begin{tabular}{llll}
\hline Compound & $\mathrm{EC}_{\mathbf{5 0}}(\mathbf{m g} / \mathbf{m L})$ & Compound & $\mathrm{EC}_{\mathbf{5 0}}(\mathbf{m g} / \mathbf{m L})$ \\
\hline $\mathbf{6 a}$ & $0.4699 \pm 0.013$ & $\mathbf{6 g}$ & $0.0827 \pm 0.017$ \\
$\mathbf{6 b}$ & $0.4967 \pm 0.015$ & $\mathbf{6 h}$ & $0.0918 \pm 0.032$ \\
$\mathbf{6 c}$ & $0.1885 \pm 0.014$ & $\mathbf{6 i}$ & $0.9434 \pm 0.018$ \\
$\mathbf{6 d}$ & $0.1720 \pm 0.018$ & $\mathbf{6 j}$ & $0.0525 \pm 0.015$ \\
$\mathbf{6 e}$ & $0.5954 \pm 0.029$ & $\mathbf{N O}_{\mathbf{2}}$-Arg-OMe & $1.8487 \pm 0.026$ \\
$\mathbf{6 f}$ & $0.4182 \pm 0.012$ & $\mathbf{L - A r g}$ & $2.0574 \pm 0.011$ \\
Vitamin E & $0.0075 \pm 0.008$ & & \\
\hline
\end{tabular}

Data are mean $\pm S D(n=3, p<0.05)$

of $\mathrm{NO}_{2}$-Arg-OMe, the corresponding compound $\mathbf{6 a}$ $\left(\mathrm{EC}_{50}=1.6235 \pm 0.015\right)$ being 1.6 times more active than $\mathrm{NO}_{2}$-Arg-OMe $\left(\mathrm{EC}_{50}=2.6169 \pm 0.032\right)$ (Table 4). Regarding the influence of radicals which substitute the phenyl ring from thiazolidine-4-one it was observed that the most favorable influence was exerted by the substitution in para with $\mathrm{Br}$, the corresponding compound $\mathbf{6 e}$, $\left(\mathrm{EC}_{50}=0.6405 \pm 0.012\right)$ being 4 times more active than the $\mathrm{NO}_{2}$-Arg-OMe. Although the activity of the all tested compounds is more intense than L-arginine, they are less active than vitamin $\mathrm{E}$ used as a positive control.

\section{Ferric reducing antioxidant power (FRAP) assay}

The ferric reducing antioxidant power assay is a sensitive method based on the reduction of ferricyanide to ferrocyanide in the presence of antioxidants with electrondonating abilities. Ferrocyanide is quantified as Perl's Prussian Blue, complex which has a maximum absorption band at $700 \mathrm{~nm}$ [36]. The absorbance values of our compounds at different concentrations $(0.56,1.13,2.27$, $4.54 \mathrm{mg} / \mathrm{mL}$ ) are shown in Fig. 4 and the $\mathrm{EC}_{50}$ values are presented in Table 5.

The derivatization of $\mathrm{NO}_{2}$-Arg-OMe through an introduction of thiazolidine-4-one moiety via amide chain has a great influence on antioxidant potential, all the tested compounds being more active than parent molecule $\left(\mathrm{NO}_{2}\right.$-Arg-OMe $)$ and $\mathrm{L}$-arginine. The most active compounds were $\mathbf{6 e}\left(\mathrm{EC}_{50}=2.5781 \pm 0.012\right)$ and $\mathbf{6 c}$ $\left(\mathrm{EC}_{50}=3.2742 \pm 0.019\right)$ which contain bromo and chloro in para position of phenyl ring. These compounds were 4.5 times and 3.4 times respectively more active than $\mathrm{NO}_{2}$-Arg-OMe $\left(\mathrm{EC}_{50}=11.0778 \pm 0.016\right)$. A good influence was produced also by substitution in meta position with methoxy and nitro, the corresponding compounds being 2.5 times $\left(\mathbf{6} \mathbf{i}, \mathrm{EC}_{50}=4.5202 \pm 0.014\right)$ and 2.4 times $\left(6 \mathrm{~g}, \mathrm{EC}_{50}=4.6474 \pm 0.018\right)$ more active than $\mathrm{NO}_{2}$-ArgOMe. All tested compounds were less active than vitamin E used as a positive control.

\section{Antibacterial/antifungal assays}

The antibacterial and antifungal activity of our derivatives was evaluated using the agar disc diffusion method and broth micro-dilution method.

\section{The agar disc diffusion method}

The data presented in Table 6 show that tested compounds are active on both bacterial and fungal strains, their effect being more intense or comparable with parent molecule $\left(\mathrm{NO}_{2}\right.$-Arg-OMe). The main characteristic of the tested compounds is their activity on $P$. aeruginosa ATCC 27853, a Gram-negative bacterial strain frequently 

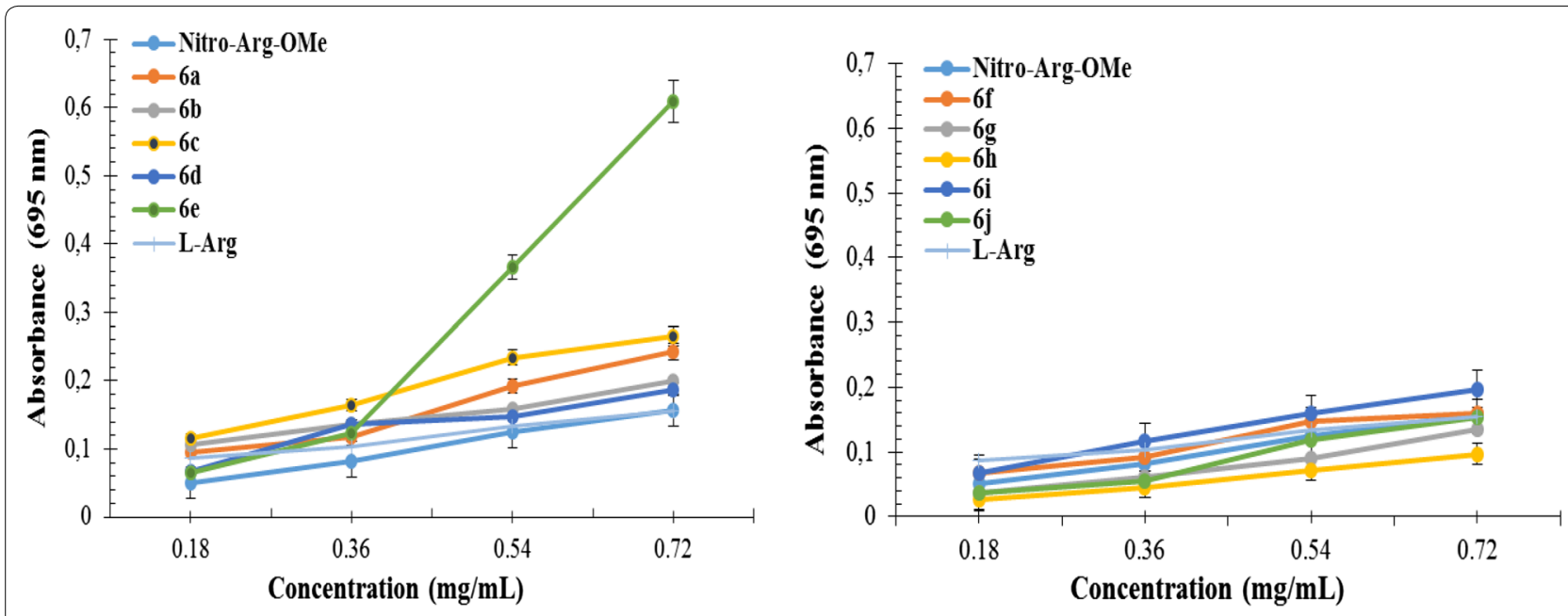

Fig. 3 The absorbance of derivatives $\mathbf{6} \mathbf{a}-\mathbf{j}$ in reference with $\mathrm{NO}_{2}-\mathrm{Arg}-\mathrm{OMe}$

Table 4 The phosphomolydenum reducing antioxidant power $\left(E C_{50} \mathrm{mg} / \mathrm{mL}\right)$ of $6 \mathrm{a}-\mathrm{j}$ derivatives

\begin{tabular}{llll}
\hline Compound & $\mathrm{EC}_{\mathbf{5 0}}(\mathbf{m g} / \mathbf{m L})$ & Compound & $\mathrm{EC}_{\mathbf{5 0}}(\mathbf{m g} / \mathbf{m L})$ \\
\hline $\mathbf{6 a}$ & $1.6235 \pm 0.015$ & $\mathbf{6 g}$ & $2.7332 \pm 0.037$ \\
$\mathbf{6 b}$ & $2.0679 \pm 0.018$ & $\mathbf{6 h}$ & $3.5186 \pm 0.018$ \\
$\mathbf{6 c}$ & $2.0734 \pm 0.022$ & $\mathbf{6 i}$ & $2.1837 \pm 0.024$ \\
$\mathbf{6 d}$ & $2.1706 \pm 0.014$ & $\mathbf{6 j}$ & $2.4610 \pm 0.019$ \\
$\mathbf{6 e}$ & $0.6405 \pm 0.012$ & $\mathbf{N O}_{\mathbf{2}}$-Arg-OMe & $2.6169 \pm 0.032$ \\
$\mathbf{6 f}$ & $2.3827 \pm 0.013$ & $\mathbf{L}-\mathbf{A r g}$ & $2.7534 \pm 0.006$ \\
Vitamin E & $0.0385 \pm 0.001$ & & \\
\hline
\end{tabular}

Data are mean $\pm S D(n=3, p<0.05)$

found in wounds. This effect is important because Gramnegative bacteria are more resistant than Gram-positive ones to the treatment due to lipopolysaccharide-rich outer membrane which significantly reduces the intracellular penetration of antibiotics [36, 37]. It is noted that in similar experimental conditions, ampicillin and chloramphenicol, used as standard drugs, were inactive on $P$. aeruginosa ATCC 27853, the data being in agreement with other experimental studies [38, 39]. The most proper compound seems to be $\mathbf{6 j}$ which has nitro in ortho position of phenyl ring. This compound was the most active against $S$. aureus, Sarcina lutea and P. aeruginosa strains in comparation with $\mathrm{NO}_{2}$-Arg-OMe (5).

Regarding the antifungal activity the data support the positive influence of nitro substitution of phenyl ring, the corresponding compounds being more active than $\mathrm{NO}_{2}$-Arg-OMe, especially on Candida albicans (6i, $\mathrm{R}=3-\mathrm{NO}_{2}, \mathbf{6 j}, \mathrm{R}=2-\mathrm{NO}_{2}$ ) and Candida glabrata (6i, $\mathbf{R}=3-\mathrm{NO}_{2}$ ). On C. glabrata a good activity was showed
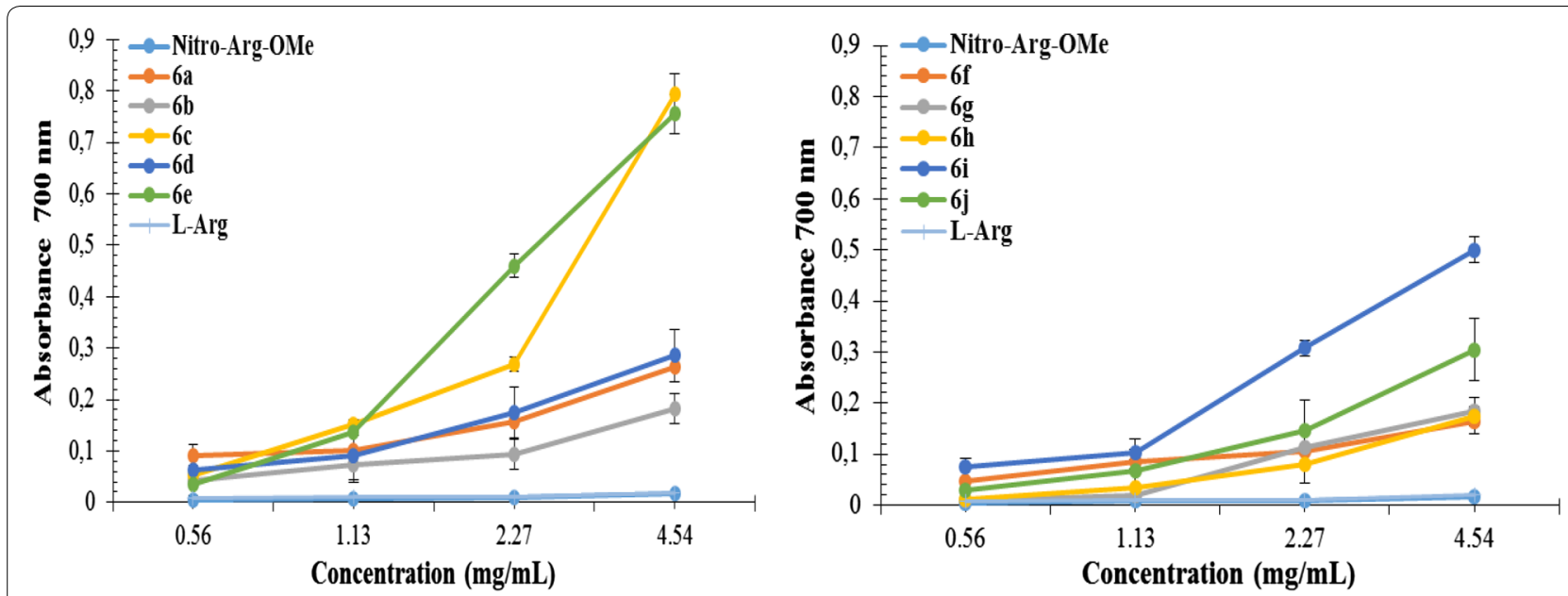

Fig. 4 The absorbance of derivatives $\mathbf{6} \mathbf{a}-\mathbf{j}$ in reference with $\mathrm{NO}_{2}-\mathrm{Arg}-\mathrm{OMe}$ 
Table 5 The ferric reducing antioxidant power $\left(\mathrm{EC}_{50}, \mathrm{mg} /\right.$ $\mathrm{mL}$ ) of $6 \mathrm{a}-\mathrm{j}$

\begin{tabular}{lllr}
\hline Compound & $\mathrm{EC}_{\mathbf{5 0}}(\mathbf{m g} / \mathbf{m L})$ & Compound & $\mathrm{EC}_{\mathbf{5 0}}(\mathbf{m g} / \mathbf{m L})$ \\
\hline $\mathbf{6 a}$ & $7.1876 \pm 0.038$ & $\mathbf{6 g}$ & $4.6474 \pm 0.018$ \\
$\mathbf{6 b}$ & $9.0695 \pm 0.015$ & $\mathbf{6 h}$ & $7.9317 \pm 0.023$ \\
$\mathbf{6 c}$ & $3.2742 \pm 0.019$ & $\mathbf{6 i}$ & $4.5202 \pm 0.014$ \\
$\mathbf{6 d}$ & $8.9671 \pm 0.023$ & $\mathbf{6 j}$ & $7.3504 \pm 0.011$ \\
$\mathbf{6 e}$ & $2.5781 \pm 0.012$ & $\mathbf{N O}_{\mathbf{2}}$-Arg-OMe & $11.0778 \pm 0.016$ \\
$\mathbf{6 f}$ & $6.1302 \pm 0.032$ & $\mathbf{L - A r g}$ & $10.9321 \pm 0.015$ \\
Vitamin E & $0.0109 \pm 0.003$ & & \\
\hline
\end{tabular}

Data are mean $\pm S D(n=3, p<0.05)$

also by $\mathbf{6 d}(\mathrm{R}=4-\mathrm{F})$. Referring to Candida parapsilosis strain it is noted that all tested compounds were more active than parent compound $\left(\mathrm{NO}_{2}\right.$-Arg-OMe, 5) and nystatin.

\section{The broth micro-dilution method}

After the antimicrobial activity was proved, the next step was to establish the minimal inhibitory concentration (MIC) and the minimal bactericidal/fungicidal concentration (MBC/MFC) using the broth micro-dilution method.

The antibacterial activity of $\mathbf{6} \mathbf{j}$ is supported by the MIC and $\mathrm{MBC}$ values (Table 7); this compound having smaller values than $\mathrm{NO}_{2}$-Arg-OMe for S. aureus and Escherichia coli. A good activity against these bacterial strains was also showed by the $\mathbf{6 c}$, which contains chloro in para position of phenyl ring of thiazolidine-4-one moiety. The data support also the antibacterial effect of $\mathbf{6 i}$ and $\mathbf{6 f}$ against $P$. aeruginosa, their $\mathrm{MIC}$ and $\mathrm{MBC}$ values being smaller than $\mathrm{NO}_{2}$-Arg-OMe.

Although the results obtained using agar disc diffusion method support that some of tested compounds are more active than positive control (ampicillin and chloramphenicol), this observation has not been proved by the MIC and MBC values. All tested compounds were less active ampicillin and chloramphenicol on tested bacterial strains, except $P$. aeruginosa ATCC 27853.

The results obtained for antifungal activity (Table 8) support the selectivity of the almost tested compounds, included the parent compound $\left(\mathrm{NO}_{2}-\mathrm{Arg}-\mathrm{OMe}\right)$, on C. parapsilosis strain. For this strain the MIC values of almost tested compounds were comparable with nystatin while the MFC values were even lower than it. The data support also the activity of $\mathbf{6} \mathbf{i}$ on C. albicans in comparation with $\mathrm{NO}_{2}$-Arg-OMe.

\section{Experimental section \\ General methods}

All chemicals used for the synthesis of the desired compounds were obtained from Sigma Aldrich Company and Fluka Company and were used as received without additional purification. The melting points were measured

Table 6 Antibacterial/antifungal inhibition area $(\mathrm{mm})$ of $6 \mathrm{a}-\mathrm{j}$ derivatives

\begin{tabular}{|c|c|c|c|c|c|c|c|}
\hline \multirow[t]{3}{*}{ Sample } & \multicolumn{7}{|c|}{ Diameter of inhibition area $^{\mathrm{a}}(\mathrm{mm})$} \\
\hline & \multicolumn{4}{|c|}{ Bacterial strains } & \multicolumn{3}{|c|}{ Yeasts strains } \\
\hline & SA & SL & EC & PA & CA & CG & $\mathrm{CP}$ \\
\hline $6 a$ & $15.2 \pm 0.12$ & $19.3 \pm 0.15$ & $10.1 \pm 0.06$ & $13.1 \pm 0.24$ & $11.8 \pm 0.35$ & $15.2 \pm 0.28$ & $23.0 \pm 0.19$ \\
\hline $6 b$ & $14.1 \pm 0.08$ & $20.1 \pm 0.13$ & $15.1 \pm 0.23$ & $11.2 \pm 0.41$ & $12.9 \pm 0.06$ & $15.2 \pm 0.98$ & $24.1 \pm 0.65$ \\
\hline $6 c$ & $15.2 \pm 0.16$ & $18.1 \pm 0.78$ & $11.2 \pm 0.63$ & $11.9 \pm 0.09$ & $9.9 \pm 0.62$ & $13.8 \pm 0.07$ & $21.2 \pm 0.33$ \\
\hline $6 d$ & $15.3 \pm 0.68$ & $18.2 \pm 0.55$ & $10.2 \pm 0.37$ & - & $13.2 \pm 0.21$ & $16.4 \pm 0.78$ & $24.2 \pm 0.35$ \\
\hline $6 e$ & $12.1 \pm 0.09$ & $20.1 \pm 0.43$ & $10.1 \pm 0.32$ & $11.1 \pm 0.19$ & $12.1 \pm 0.58$ & $15.9 \pm 0.55$ & $25.3 \pm 0.28$ \\
\hline $6 f$ & $15.2 \pm 0.52$ & $20.1 \pm 0.26$ & $12.2 \pm 1.05$ & $10.2 \pm 0.36$ & $12.1 \pm 0.18$ & $15.5 \pm 0.48$ & $25.1 \pm 0.37$ \\
\hline $6 g$ & $13.1 \pm 0.15$ & $20.1 \pm 0.72$ & - & $10.1 \pm 0.09$ & $12.1 \pm 0.28$ & $15.9 \pm 1.07$ & $25.2 \pm 0.39$ \\
\hline $6 h$ & $14.1 \pm 0.09$ & $20.3 \pm 0.43$ & $11.1 \pm 0.30$ & $10.2 \pm 0.15$ & $12.1 \pm 0.86$ & $13.8 \pm 0.57$ & $23.1 \pm 0.22$ \\
\hline $6 i$ & $12.3 \pm 0.08$ & $21.1 \pm 0.13$ & $10.1 \pm 0.23$ & $12.2 \pm 0.41$ & $15.4 \pm 0.06$ & $16.4 \pm 0.98$ & $20.1 \pm 0.65$ \\
\hline $\mathbf{6 j}$ & $16.3 \pm 0.34$ & $21.2 \pm 0.87$ & $10.2 \pm 0.51$ & $13.1 \pm 0.82$ & $15.2 \pm 0.74$ & $15.2 \pm 0.32$ & $23.1 \pm 0.47$ \\
\hline 5 & $14.9 \pm 0.16$ & $19.9 \pm 0.12$ & $11.9 \pm 0.06$ & $11.8 \pm 0.19$ & $13.8 \pm 0.15$ & $15.9 \pm 0.17$ & $19.9 \pm 0.09$ \\
\hline A & $20.1 \pm 0.57$ & $21.2 \pm 1.16$ & $15.2 \pm 0.67$ & - & - & - & - \\
\hline C & $16.3 \pm 0.28$ & $30.4 \pm 0.35$ & $20.1 \pm 0.16$ & - & - & - & - \\
\hline $\mathbf{N}$ & - & - & - & - & $19.4 \pm 0.51$ & $19.5 \pm 0.72$ & $12.4 \pm 0.42$ \\
\hline
\end{tabular}

SA = Staphylococcus aureus ATCC 25923; SL = Sarcina lutea ATCC 9341; EC = Escherichia coli ATCC 25922; PA = Pseudomonas aeruginosa ATCC 27853; CA = Candida albicans ATCC 10231; CG = Candida glabrata ATCC MYA 2950; CP = Candida parapsilosis ATCC 22019; $5=\mathrm{NO}_{2}$-Arg-OMe; $\mathrm{A}=$ ampicillin; $\mathrm{C}=$ chloramphenicol; $\mathrm{N}=$ nystatin. $\mathbf{5}=\mathrm{L}_{-} \mathrm{NO}_{2}$-Arg-OMe

a Mean values $(\mathrm{n}=3) \pm$ standard deviation 
Table 7 Antibacterial effect expressed as MIC and MBC values $(\mathrm{mg} / \mathrm{mL})$ of $6 a-j$

\begin{tabular}{|c|c|c|c|c|c|c|c|c|}
\hline \multirow[t]{2}{*}{ Sample } & \multicolumn{2}{|c|}{$\begin{array}{l}\text { S. aureus } \\
\text { ATCC } 25923\end{array}$} & \multicolumn{2}{|c|}{$\begin{array}{l}\text { S. lutea } \\
\text { ATCC } 9341\end{array}$} & \multicolumn{2}{|c|}{$\begin{array}{l}\text { E. coli } \\
\text { ATCC } 25922\end{array}$} & \multicolumn{2}{|c|}{$\begin{array}{l}\text { P. aeruginosa } \\
\text { ATCC } 27853\end{array}$} \\
\hline & $\mathrm{MIC}^{\mathrm{a}}$ & $\overline{M B C^{a}}$ & $\mathrm{MIC}^{\mathrm{a}}$ & $\mathrm{MBC}^{\mathrm{a}}$ & $\overline{M I C^{a}}$ & $\overline{M B C^{a}}$ & $\overline{\mathrm{MIC}^{\mathrm{a}}}$ & $\overline{M B C^{a}}$ \\
\hline $6 a$ & 2.5 & 2.5 & 0.01 & 0.01 & 1.25 & 1.25 & 2.5 & 2.5 \\
\hline $6 b$ & 1.25 & 2.5 & 0.03 & 1.25 & 2.5 & 10 & 2,5 & 10 \\
\hline $6 c$ & 0.01 & 0.01 & 0.01 & 0.03 & 0.03 & 0.03 & 5 & 5 \\
\hline $6 d$ & 2.5 & 2.5 & 0.01 & 0.03 & 1.25 & 5 & 1.25 & 1.25 \\
\hline $6 e$ & 0.01 & 0.3 & 0.01 & 0.01 & 10 & 5 & 5 & 5 \\
\hline $6 f$ & 0.07 & 2.5 & 0.03 & 0.15 & 0.03 & 0.15 & 0.03 & 1.25 \\
\hline $6 \mathrm{~g}$ & 2.5 & 2.5 & 0.03 & 1.25 & 1.25 & 1.25 & 2.5 & 10 \\
\hline $6 \mathrm{~h}$ & 2.5 & 2.5 & 0.01 & 0.01 & 1.25 & 5 & 2.5 & 2.5 \\
\hline $6 i$ & 0.3 & 0.3 & 0.01 & 0.01 & 0.1 & 0.1 & 0.03 & 0.6 \\
\hline $6 \mathbf{j}$ & 0.07 & 0.07 & 0.01 & 0.01 & 0.03 & 0.03 & 1.25 & 1.25 \\
\hline 5 & 2.5 & 2.5 & 0.01 & 0.01 & 1.25 & 1.25 & 1.25 & 1.25 \\
\hline A & 0.0002 & 0.0005 & 0.0002 & 0.0005 & 0.008 & 0.016 & $\mathrm{nt}$ & nt \\
\hline C & 0.008 & 0.016 & 0.003 & 0.006 & 0.008 & 0.016 & $\mathrm{nt}$ & $\mathrm{nt}$ \\
\hline
\end{tabular}

$\mathbf{5}=\mathrm{L}_{-} \mathrm{NO}_{2}$-Arg-OMe, $\mathrm{A}=$ ampicillin; $\mathrm{C}=$ chloramphenicol; $\mathrm{nt}=$ no tested

a Mean values $(n=3) \pm$ standard deviation

Table 8 Antifungal effect expressed as MIC and MFC values $(\mathrm{mg} / \mathrm{mL})$ of $6 a-j$

\begin{tabular}{|c|c|c|c|c|c|c|}
\hline \multirow[t]{2}{*}{ Sample } & \multicolumn{2}{|c|}{$\begin{array}{l}\text { C. albicans } \\
\text { ATCC } 10231\end{array}$} & \multicolumn{2}{|c|}{$\begin{array}{l}\text { C. glabrata } \\
\text { ATCC MYA } 2950\end{array}$} & \multicolumn{2}{|c|}{$\begin{array}{l}\text { C. parapsilosis } \\
\text { ATCC } 22019\end{array}$} \\
\hline & $\mathrm{MIC}^{\mathrm{a}}$ & $\overline{M F C^{a}}$ & $\mathrm{MIC}^{\mathrm{a}}$ & $\overline{M F C^{a}}$ & $\mathrm{MIC}^{\mathrm{a}}$ & $\overline{M F C^{a}}$ \\
\hline $6 a$ & 0.6 & 1.25 & 1.25 & 1.25 & 0.003 & 0.003 \\
\hline $6 b$ & 0.6 & 1.25 & 2.5 & 10 & 0.003 & 0.003 \\
\hline $6 c$ & 0.6 & 0.6 & 10 & 10 & 0.003 & 0.003 \\
\hline $6 d$ & 0.3 & 1.25 & 0.6 & 2.5 & 0.003 & 0.003 \\
\hline $6 e$ & 10 & 10 & 10 & 10 & 5 & 5 \\
\hline $6 f$ & 0.6 & 0.6 & 2.5 & 5 & 0.003 & 0.003 \\
\hline $6 \mathrm{~g}$ & 0.3 & 1.25 & 1.25 & 2.5 & 0.003 & 0.003 \\
\hline $6 \mathrm{~h}$ & 0.3 & 5 & 10 & 10 & 0.003 & 0.003 \\
\hline $6 \mathbf{i}$ & 0.03 & 10 & 10 & 10 & 10 & 10 \\
\hline $6 j$ & 0.3 & 1.25 & 1.25 & 1.25 & 0.003 & 0.003 \\
\hline 5 & 1.25 & 1.25 & 0.6 & 2.5 & 0.003 & 0.003 \\
\hline $\mathbf{N}$ & 0.004 & 0.008 & 0.004 & 0.008 & 0.004 & 0.008 \\
\hline
\end{tabular}

$\mathbf{5}=-\mathrm{L}-\mathrm{NO}_{2}-$ Arg-OMe, $\mathrm{N}=$ nystatin

a Mean values $(n=3) \pm$ standard deviation

using a Buchi Melting Point B-540 apparatus and they are uncorrected. The FT-IR spectra were recorded on Horizon MBTM FT-IR, over a $500-4000 \mathrm{~cm}^{-1}$ range, after 16 scans at a resolution of $4 \mathrm{~cm}^{-1}$. The spectra processing was carried out with the Horizon MBTM FTIR Software. The ${ }^{1} \mathrm{H}$-NMR $(400 \mathrm{MHz})$ and ${ }^{13} \mathrm{C}-\mathrm{NMR}(101 \mathrm{MHz})$ spectra were obtained on a Bruker Avance $400 \mathrm{MHz}$ spectrometer using tetramethylsilane as internal standard and deuterated chloroform as solvent $\left(\mathrm{CDCl}_{3}\right)$. The chemical shifts were shown in $\delta$ values (ppm). The mass spectra were registered using a Bruker MaXis Ultra-High Resolution Quadrupole Time-of-Flight Mass Spectrometer. The progress of the reaction was monitored on TLC, using pre-coated Kieselgel 60 F254 plates (Merck, Whitehouse Station, NJ, USA) and the compounds were visualized using UV light. E-factor and material efficiency (ME) have been selected to evaluate the greenness of the synthetic procedures. E-factor is a very useful metric tool that is defined as E-Factor $=$ mass of wastes/mass of product. The E-factor can be used to calculate the 
material efficiency of the process according to the equation: $\mathrm{ME}=1 / \mathrm{E}$-factor +1 [40].

The antioxidant potential was investigated using in vitro methods based on ferric/phosphomolybdenum reducing antioxidant power and DPPH/ABTS radical scavenging assay. The antibacterial activity was evaluated using Grampositive (S. aureus ATCC 25923, S. lutea ATCC 9341) and Gram-negative (E. coli ATCC 25922 and P. aeruginosa ATCC 27853) bacterial strains. The antifungal activity was evaluated using C. albicans ATCC 10231, C. glabrata ATCC MYA 2950 and C. parapsilosis ATCC 22019. All strains were obtained from the Culture Collection of the Department of Microbiology, Gr. T. Popa University of Medicine and Pharmacy, Iasi, Romania. As positive controls were used ampicillin, a beta-lactam drug, and chloramphenicol which belongs amphenicoles class for antibacterial activity and nystatin for antifungal activity.

\section{General procedure for synthesis \\ of $N^{2}$-[(2-aryl-4-oxo-1,3-thiazolidin-3-yl) \\ propionyl]-nitro-L-arginine methyl ester $(6 \mathbf{a}-\mathbf{j})$}

3-(2-Phenyl-4-oxo-1,3-thiazolidin-3-yl)propionic acid derivatives, $4 \mathbf{a}-\mathbf{j}(5 \mathrm{mmol})$ were dissolved in $25 \mathrm{~mL}$ freshly distilled DCM, on ice bath at $0-5{ }^{\circ} \mathrm{C}$ and under inert atmosphere of nitrogen [41]. To the cold solution it was added EDCI. $\mathrm{HCl}$ (5.5 mmol, 1.1 equiv.), HOBt (5.5 mmol, 1.1 equiv.) and $\mathrm{NO}_{2}$-L-Arg-OMe. $\mathrm{HCl}(5.5 \mathrm{mmol}, 1.1$ equiv.). The mixture was stirred for $10-14 \mathrm{~h}$ at room temperature. The reaction monitoring was carried out by Thin Layer Chromatography (TLC) using as mobile phase DCM: methanol $(\mathrm{MeOH})=9.5$ : $0.5(\mathrm{v} / \mathrm{v})$ and the spot visualization was done under UV light at $254 \mathrm{~nm}$. After the completion of the reaction, the mixture was washed successively with $1 \mathrm{M} \mathrm{HCl}$, saturated solution of sodium bicarbonate and saturated brine solution. The organic layer, was dried over anhydrous $\mathrm{MgSO}_{4}$, filtered and concentrated to dryness. Purification of compounds was carried out by column separation on silica gel (DCM/MeOH, 9.5/0.5). The appropriate fractions of thiazolidine-4-one derivatives was collected and then evaporated to dryness to give the corresponding final derivatives.

\section{$N^{2}$-[(2-Phenyl-4-oxo-1,3-thiazolidin-3-yl)}

\section{propionyl]-nitro-L-arginine methyl ester (6a)}

White cristals, mp $102{ }^{\circ} \mathrm{C}$, yield: $93 \%$, IR (Zn/Se crystal, $\left.\mathrm{cm}^{-1}\right)$ : $3294(-\mathrm{NH}) ; 2963,783\left(=\mathrm{CH}_{\text {phenyl }}\right) ; 2869,1250$, $725\left(-\mathrm{CH}_{2}-\right) ; 1736\left(\mathrm{COOCH}_{3}\right) ; 1647(\mathrm{CONH}) ; 1628$ $\left(\mathrm{C}=\mathrm{O}_{\text {thiazolidine-4-one }}\right) ; 1535 \quad\left(-\mathrm{C}=\mathrm{C}-{ }_{\text {phenyl }}\right) ; 1350,1026$ $(-\mathrm{C}-\mathrm{N}-)$; $698(\mathrm{C}-\mathrm{S}) ;{ }^{1} \mathrm{H}-\mathrm{NMR}(\delta \mathrm{ppm}): 8.51(\mathrm{~s}, 1 \mathrm{H}, \mathrm{N} \underline{\mathrm{H}}-$ $\mathrm{CO}), 8.03(\mathrm{~m}, 1 \mathrm{H}, \mathrm{NH}), 7.56-7.47(\mathrm{~m}, 2 \mathrm{H}, \mathrm{N} \underline{\mathrm{H}}), 7.38-$ $7.29(\mathrm{~m}, 5 \mathrm{H}, \mathrm{Ar}-\underline{\mathrm{H}}), 5.77(\mathrm{~d}, \mathrm{~J}=55.7 \mathrm{~Hz}, 1 \mathrm{H},-\mathrm{N}-\mathrm{C} \underline{\mathrm{H}}-\mathrm{S})$, 4.61 (s, $1 \mathrm{H}, \mathrm{CH}_{\underline{2}}-\mathrm{S}$ ), 3.89 (s, $\left.1 \mathrm{H}, \mathrm{CH}_{\underline{2}}-\mathrm{S}\right), 3.78$ (s, $3 \mathrm{H}, \mathrm{C}_{\underline{3}}$ ester), $3.73\left(\mathrm{~s}, 1 \mathrm{H}, \mathrm{C} \underline{\mathrm{H}}-\mathrm{COOCH}_{3}\right), 3.69\left(\mathrm{~s}, 1 \mathrm{H}, \mathrm{N}-\mathrm{CH}_{2}\right)$, 3.39-3.30 (m, $\left.2 \mathrm{H}, \mathrm{C}_{2} \mathrm{arg}\right), 3.23-3.01\left(\mathrm{~m}, 1 \mathrm{H}, \mathrm{N}-\mathrm{CH}_{2}\right.$ ), 2.62-2.34 (m, $\left.2 \mathrm{H}, \mathrm{C}_{2}-\mathrm{CO}\right), 1.94-1.54\left(\mathrm{~m}, 4 \mathrm{H}, 2 \underline{\mathrm{C}}_{2}\right.$ $\arg ) ;{ }^{13} \mathrm{C}-\mathrm{NMR}(\delta \mathrm{ppm}): 172.32,171.28,162.09$ (3C, $\left.\underline{\mathrm{CO}}\right)$, 159.64 $\left(\mathrm{C}_{\text {guanid }}\right), 139.15,129.72,129.42,127.45,127.33$, $117.60\left(6 \mathrm{C}, \mathrm{C}_{\mathrm{Ar}}\right), 64.36(\mathrm{~S}-\underline{\mathrm{C}} \mathrm{H}-\mathrm{N}-), 52.99\left(\mathrm{CH}_{2}\right), 48.47$ $\left.(\underline{\mathrm{C}} \mathrm{H}), 39.75\left(-\underline{\mathrm{CH}}_{2} \mathrm{~N}-\right), 33.99\left(-\underline{\mathrm{CH}}_{2} \mathrm{~S}-\right), 33.33(\underline{\mathrm{CH}})_{2}\right)$, $32.98\left(-\mathrm{CH}_{2} \mathrm{CO}\right), 24.29\left(\mathrm{CH}_{2}\right), 20.57\left(\mathrm{CH}_{3}\right) ;$ HRMS (EI-MS): $\mathrm{m} / z$ calculated for $\mathrm{C}_{19} \mathrm{H}_{26} \mathrm{~N}_{6} \mathrm{O}_{6} \mathrm{~S}[\mathrm{M}+\mathrm{H}]^{+}$ 467.1707; found is 467.1705; Green chemistry metrics: E-factor 22.513, ME 0.042 .

\section{$N^{2}$-[(2-(4-Methylphenyl)-4-oxo-1,3-thiazolidin-3-yl) propionyl]-nitro-L-arginine methyl ester (6b)}

Light yellow cristals, mp $90{ }^{\circ} \mathrm{C}$, yield: $91 \%$, IR ( $\mathrm{Zn} /$ Se crystal, cm $\left.{ }^{-1}\right)$ : $3305(-\mathrm{NH})$; 2951, $771\left(=\mathrm{C}-\mathrm{H}_{\text {phe- }}\right.$ nyl); 2928, 1257, $721\left(-\mathrm{CH}_{2}-\right) ; 1724\left(\mathrm{COOCH}_{3}\right) ; 1678$ $(\mathrm{CONH}) ; 1628\left(\mathrm{C}=\mathrm{O}_{\text {thiazolidine-4-one }}\right) ; 1597 \quad\left(-\mathrm{C}=\mathrm{C}-{ }_{\text {phe- }}\right.$ $\left.{ }_{\text {nyl }}\right) ; 1362,1026$ (-C-N-); $694(\mathrm{C}-\mathrm{S}) ;{ }^{1} \mathrm{H}-\mathrm{NMR}(\delta \mathrm{ppm})$ : $8.68(\mathrm{~s}, 1 \mathrm{H}, \mathrm{N} \underline{\mathrm{H}}-\mathrm{CO}), 8.31(\mathrm{~m}, 1 \mathrm{H}, \mathrm{NH}), 7.80(\mathrm{~s}, 2 \mathrm{H}$, $\mathrm{NH}), 7.26-7.34(\mathrm{~m}, 4 \mathrm{H}, \mathrm{Ar}-\underline{\mathrm{H}}), 5.82(\mathrm{~d}, \mathrm{~J}=18.8 \mathrm{~Hz}$, $1 \mathrm{H},-\mathrm{N}-\mathrm{C} \underline{\mathrm{H}}-\mathrm{S}$ ), $4.57\left(\mathrm{~s}, 1 \mathrm{H}, \mathrm{CH}_{\underline{2}}-\mathrm{S}\right), 3.92$ (dd, $\mathrm{J}=13.6$, $\left.6.8 \mathrm{~Hz}, 1 \mathrm{H}, \mathrm{CH}_{2}-\mathrm{S}\right), 3.81\left(\mathrm{~s}, 3 \mathrm{H}, \mathrm{C}_{3}\right.$ ester), $3.78(\mathrm{~s}, 1 \mathrm{H}$, $\left.\mathrm{C} \underline{\mathrm{H}}-\mathrm{COOCH}_{3}\right), 3.71\left(\mathrm{~s}, 1 \mathrm{H}, \mathrm{N}-\mathrm{CH}_{2}\right), 3.53-3.31(\mathrm{~m}, 2 \mathrm{H}$, $\mathrm{CH}_{2}$ arg), 3.24-3.05 (m, 1H, N- $\left.\mathrm{CH}_{2}\right), 2.62$ (dd, J = 18.0, $\left.7.9 \mathrm{~Hz}, 2 \mathrm{H}, \mathrm{CH}_{2}-\mathrm{CO}\right), 2.41\left(\mathrm{~s}, 3 \mathrm{H}, \mathrm{CH}_{3}\right), 2.02-1.62(\mathrm{~m}$, $\left.4 \mathrm{H}, 2 \underline{\mathrm{H}}_{2} \arg \right) ;{ }^{13} \mathrm{C}-\mathrm{NMR}(\delta \mathrm{ppm}): 172.47,171.28,170.76$ $(3 \mathrm{C}, \underline{\mathrm{CO}}), 159.40\left(\mathrm{C}_{\text {guanid }}\right), 138.24,134.57\left(2 \mathrm{C}, \mathrm{C}_{\mathrm{Ar}}\right)$, $128.95\left(2 \mathrm{C}, \underline{\mathrm{C}} \mathrm{H}_{\mathrm{Ar}}\right), 123.31\left(2 \mathrm{C}, \underline{\mathrm{C}}_{\mathrm{Ar}}\right), 63.25(\mathrm{~S}-\underline{\mathrm{C}} \mathrm{H}-$ $\mathrm{N}), 50.34(\underline{\mathrm{CH}}), 40.73\left(\underline{\mathrm{CH}}_{2}\right), 39.58\left(-\underline{\mathrm{CH}}_{2} \mathrm{~N}-\right), 33.45$ $\left(-\underline{\mathrm{CH}}_{2} \mathrm{~S}-\right)$, 32.84 $\left(-\mathrm{CH}_{2} \mathrm{CO}\right), 29.14\left(\underline{\mathrm{CH}}_{2}\right), 24.29\left(\underline{\mathrm{CH}}_{2}\right)$, 26.37, 21.34 (2C, $\left.\mathrm{CH}_{3}\right)$; HRMS (EI-MS): $\mathrm{m} / z$ calculated for $\mathrm{C}_{20} \mathrm{H}_{28} \mathrm{~N}_{6} \mathrm{O}_{6} \mathrm{~S}[\mathrm{M}+\mathrm{H}]^{+}$481.1862; found 481.1864; Green chemistry metrics: E-factor 16.891, ME 0.056.

\section{$N^{2}$-[(2-(4-Chlorophenyl)-4-oxo-1,3-thiazolidin-3-yl) propionyl]-nitro-L-arginine methyl ester $(6 \mathrm{c})$}

Light yellow cristals, mp $146{ }^{\circ} \mathrm{C}$, yield: 89 \%; IR (Zn/Se crystal, $\left.\mathrm{cm}^{-1}\right)$ : $3302(-\mathrm{NH})$; 2951, $783\left(=\mathrm{C}-\mathrm{H}_{\text {phenyl }}\right)$; 2928, 1257, $725\left(-\mathrm{CH}_{2}-\right)$; $1736\left(\mathrm{COOCH}_{3}\right) ; 1651$ ($\mathrm{CONH}) ; 1628\left(\mathrm{C}=\mathrm{O}_{\text {thiazolidine-4-one }}\right) ; 1597\left(-\mathrm{C}=\mathrm{C}-{ }_{\text {phenyl }}\right)$; 1342, 1014 (-C-N-);764 (C-Cl); 683 (C-S); ${ }^{1} \mathrm{H}-\mathrm{NMR}$

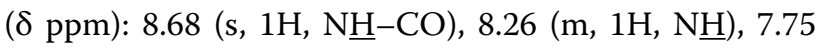
(s, $2 \mathrm{H}, \mathrm{NH}), 7.32(\mathrm{~d}, \mathrm{~J}=8.2 \mathrm{~Hz}, 2 \mathrm{H}, \mathrm{Ar}-\mathrm{H}), 7.28-7.23$ (d, $2 \mathrm{H}, \mathrm{Ar}-\mathrm{H}), 5.75$ (d, J = $26.3 \mathrm{~Hz}, 1 \mathrm{H},-\mathrm{N}-\mathrm{C} \underline{\mathrm{H}}-\mathrm{S})$, 4.52 (s, $\left.1 \mathrm{H}, \mathrm{CH}_{\underline{2}}-\mathrm{S}\right), 3.79$ (dd, J = 15.8, 8.6 Hz, 1H, C$\mathrm{COOCH}_{3}$ ), 3.71 (s, $\left.1 \mathrm{H}, \mathrm{CH}_{2}-\mathrm{S}\right), 3.68\left(\mathrm{~s}, 3 \mathrm{H}, \mathrm{C}_{3}\right.$ ester), $3.64\left(\mathrm{~s}, 1 \mathrm{H}, \mathrm{N}-\mathrm{CH}_{2}\right), 3.31$ (d, J = 44.9 Hz, $\left.2 \mathrm{H}, \underline{\mathrm{C}}_{2} \arg \right)$, 3.11-2.94 (m, $\left.1 \mathrm{H}, \mathrm{N}-\mathrm{CH}_{2}\right), 2.65-2.29\left(\mathrm{~m}, 2 \mathrm{H}, \mathrm{CH}_{2}-\right.$ $\mathrm{CO}), 1.90-1.56\left(\mathrm{~m}, 4 \mathrm{H}, 2 \mathrm{C}_{2} \mathrm{arg}\right) ;{ }^{13} \mathrm{C}-\mathrm{NMR}(\delta \mathrm{ppm})$ : 173.24, 171.99, 169.52 (3C, $\underline{\mathrm{CO}}), 160.48$ ( $\left.\mathrm{C}_{\text {guanid }}\right), 138.42$, $135.80\left(\mathrm{C}_{\mathrm{Ar}}\right), 130.05\left(2 \mathrm{C}, \underline{\mathrm{C}} \mathrm{H}_{\mathrm{Ar}}\right), 129.37\left(2 \mathrm{C}, \underline{\mathrm{C}}_{\mathrm{Ar}}\right), 63.94$ (S- $\underline{\mathrm{C}} \mathrm{H}-\mathrm{N}), 53.38\left(\underline{\mathrm{CH}}_{2}\right), 51.34(\underline{\mathrm{C}} \mathrm{H}), 41.42\left(\underline{\mathrm{CH}}_{2}\right), 39.10$ 
$\left(-\underline{\mathrm{CH}}_{2} \mathrm{~N}-\right), 34.19\left(-\mathrm{C}_{2} \mathrm{~S}-\right), 31.53\left(-\mathrm{C}_{2} \mathrm{CO}\right), 29.57$ $\left(\mathrm{CH}_{2}\right) ; 26.45\left(\mathrm{CH}_{3}\right) ;$ HRMS (EI-MS): $\mathrm{m} / z$ calculated for $\mathrm{C}_{19} \mathrm{H}_{25} \mathrm{ClN}_{6} \mathrm{O}_{6} \mathrm{~S}[\mathrm{M}+\mathrm{H}]^{+}$501.1317; found 501.1310; Green chemistry metrics: E-factor 2.361, ME 0.297.

\section{$N^{2}$-[(2-(4-Fluorophenyl)-4-oxo-1,3-thiazolidin-3-yl) propionyl]-nitro-L-arginine methyl ester (6d)}

Light yellow cristals, mp $85{ }^{\circ} \mathrm{C}$, yield: $75 \%$; IR ( $\mathrm{Zn} / \mathrm{Se}$ crystal, $\left.\mathrm{cm}^{-1}\right)$ : $3302(-\mathrm{NH})$; 2951, $787\left(=\mathrm{C}-\mathrm{H}_{\text {phenyl }}\right)$; 2933, 1257, $725\left(-\mathrm{CH}_{2}-\right)$; $1736\left(\mathrm{COOCH}_{3}\right) ; 1651$ ($\mathrm{CONH}) ; 1647\left(\mathrm{C}=\mathrm{O}_{\text {thiazolidine-4-one }}\right) ; 1601\left(-\mathrm{C}=\mathrm{C}-{ }_{\text {phenyl }}\right)$; 1342, 1011 (-C-N-); 1153 (C-F); 687 (C-S); ${ }^{1} \mathrm{H}-\mathrm{NMR}$ $(\delta \mathrm{ppm}): 8.63(\mathrm{~s}, 1 \mathrm{H}, \mathrm{N} \underline{\mathrm{H}}-\mathrm{CO}), 8.24(\mathrm{~m}, 1 \mathrm{H}, \mathrm{N} \underline{\mathrm{H}}), 7.61$ (s, $2 \mathrm{H}, \mathrm{NH}$ ), 7.37 (dd, J = 13.7, $5.7 \mathrm{~Hz}, 2 \mathrm{H}, \mathrm{Ar}-\mathrm{H}), 7.11$ $(\mathrm{t}, \mathrm{J}=8.4 \mathrm{~Hz}, 2 \mathrm{H}, \mathrm{Ar}-\mathrm{H}), 5.80(\mathrm{~d}, \mathrm{~J}=50.0 \mathrm{~Hz}, 1 \mathrm{H},-\mathrm{N}-$ C$-\mathrm{S}), 4.72-4.41\left(\mathrm{~m}, 1 \mathrm{H}, \mathrm{CH}_{2}-\mathrm{S}\right), 3.94-3.85(\mathrm{~m}, 1 \mathrm{H}$, C트-COOCH$\left.{ }_{3}\right), 3.80\left(\mathrm{~s}, 1 \mathrm{H}, \mathrm{CH}_{2}-\mathrm{S}\right), 3.75\left(\mathrm{~s}, 3 \mathrm{H}, \mathrm{CH}_{3}\right.$ ester), $3.71\left(\mathrm{~s}, 1 \mathrm{H}, \mathrm{N}-\mathrm{CH}_{2}\right), 3.52-3.27\left(\mathrm{~m}, 2 \mathrm{H}, \mathrm{CH}_{2} \mathrm{arg}\right)$, 3.20-3.00 (m, $1 \mathrm{H}, \mathrm{N}-\mathrm{CH}_{2}$ ), 2.68-2.27 (m, $\left.2 \mathrm{H}, \mathrm{CH}_{2}-\mathrm{CO}\right)$, 1.84-1.59 (m, $4 \mathrm{H}, 2 \mathrm{CH}_{2}$ arg); ${ }^{13} \mathrm{C}-\mathrm{NMR}$ ( $\left.\delta \mathrm{ppm}\right):$ 170.76, 162.34 (3C, $\underline{\mathrm{CO}}), 158.81\left(\mathrm{C}_{\text {guanid }}\right), 161.58,135.57$ $\left(2 \mathrm{C}, \mathrm{C}_{\mathrm{Ar}}\right), 128.32\left(2 \mathrm{C}, \underline{\mathrm{C}} \mathrm{H}_{\mathrm{Ar}}\right), 115.61\left(2 \mathrm{C}, \underline{\mathrm{C}} \mathrm{H}_{\mathrm{Ar}}\right), 62.65$ $(\mathrm{S}-\mathrm{CH}-\mathrm{N}), 52.15(\underline{\mathrm{CH}}), 39.93\left(-\mathrm{CH}_{2} \mathrm{~N}-\right), 38.85\left(\mathrm{CH}_{2}\right)$, $33.10\left(-\underline{\mathrm{CH}}_{2} \mathrm{~S}-\right), 32.16\left(-\underline{\mathrm{CH}}_{2} \mathrm{CO}\right), 29.24\left(\underline{\mathrm{CH}}_{2}\right), 28.67$ $\left(\mathrm{CH}_{2}\right), 21.34\left(\mathrm{CH}_{3}\right) ;$ HRMS (EI-MS): $\mathrm{m} / z$ calculated for $\mathrm{C}_{19} \mathrm{H}_{25} \mathrm{FN}_{6} \mathrm{O}_{6} \mathrm{~S}[\mathrm{M}+\mathrm{H}]^{+}$485.1614; found 485.1613; Green chemistry metrics: E-factor 1.122, ME 0.471.

\section{$N^{2}$-[(2-(4-Bromophenyl)-4-oxo-1,3-thiazolidin-3-yl) propionyl]-nitro-L-arginine methyl ester (6e)}

Light yellow cristals, mp $109{ }^{\circ} \mathrm{C}$, yield: $87 \%$; IR (Zn/Se crystal, $\left.\mathrm{cm}^{-1}\right)$ : $3294(-\mathrm{NH}) ; 2954,776$ (=C-H $\left.\mathrm{H}_{\text {phenyl }}\right)$; $1736\left(\mathrm{COOCH}_{3}\right) ; 1647(-\mathrm{CONH}) ; 1628\left(\mathrm{C}=\mathrm{O}_{\text {thiazolidine- }}\right.$ 4-one); 1601 (-C=C- ${ }_{\text {phenyl }}$; 1342, 1007 (-C-N-); 1246, $725\left(-\mathrm{CH}_{2}-\right)$; $687(\mathrm{C}-\mathrm{S}) ; 668(\mathrm{C}-\mathrm{Br}) ;{ }^{1} \mathrm{H}-\mathrm{NMR}(\delta \mathrm{ppm})$ : $8.68(\mathrm{~s}, 1 \mathrm{H}, \mathrm{N} \underline{\mathrm{H}}-\mathrm{CO}), 8.19(\mathrm{~m}, 1 \mathrm{H}, \mathrm{N} \underline{\mathrm{H}}), 7.76(\mathrm{~s}, 2 \mathrm{H}$, $\mathrm{N} \underline{\mathrm{H}}), 7.54$ (d, J = 7.6 Hz, $2 \mathrm{H}, \mathrm{Ar}-\underline{\mathrm{H}}), 7.36-7.16(\mathrm{~m}, 2 \mathrm{H}$, $\mathrm{Ar}-\underline{\mathrm{H}}), 5.79$ (d, J = $29.5 \mathrm{~Hz}, 1 \mathrm{H},-\mathrm{N}-\mathrm{C} \underline{\mathrm{H}}-\mathrm{S}), 4.59$ (s, $1 \mathrm{H}$, $\mathrm{CH}_{2}-\mathrm{S}$ ), 3.86 (dd, J = 17.6, $10.2 \mathrm{~Hz}, 1 \mathrm{H}, \mathrm{CH}_{2}-\mathrm{S}$ ), 3.78 (s, $3 \mathrm{H}, \mathrm{C}_{\underline{3}}$ ester), 3.77 (s, $1 \mathrm{H}, \mathrm{C} \underline{\mathrm{H}}-\mathrm{COOCH}_{3}$ ), 3.72 (d, $\left.\mathrm{J}=15.6 \mathrm{~Hz}, 1 \mathrm{H}, \mathrm{N}-\mathrm{CH}_{2}\right), 3.37\left(\mathrm{~d}, \mathrm{~J}=45.7 \mathrm{~Hz}, 2 \mathrm{H}, \mathrm{CH}_{2}\right.$ arg), 3.09 (dd, J = 31.1, $10.2 \mathrm{~Hz}, 1 \mathrm{H}, \mathrm{N}-\mathrm{CH}_{2}$ ), 2.74-2.51 (m, 1H, $\mathrm{CH}_{2}-\mathrm{CO}$ ), 2.43-2,37 (m, 1H, $\left.\underline{\mathrm{C}}_{2}-\mathrm{CO}\right), 1.82$ (d, $\mathrm{J}=78.0 \mathrm{~Hz}, 4 \mathrm{H}, 2 \mathrm{CH}_{2}$ arg $) ;{ }^{13} \mathrm{C}-\mathrm{NMR}(\delta \mathrm{ppm}): 172.47$, 170.18, 161.28 (3C, $\underline{\mathrm{CO}}), 159.40\left(\mathrm{C}_{\text {guanid }}\right), 138.24,132.34$ $\left(2 \mathrm{C}, \mathrm{C}_{\mathrm{Ar}}\right), 128.95\left(2 \mathrm{C}, \underline{\mathrm{CH}}_{\mathrm{Ar}}\right), 123.31\left(2 \mathrm{C}, \underline{\mathrm{C}} \mathrm{H}_{\mathrm{Ar}}\right), 63.25$ $(\mathrm{S}-\underline{\mathrm{C}} \mathrm{H}-\mathrm{N}), 52.71(\underline{\mathrm{CH}}), 40.73\left(\underline{\mathrm{CH}}_{2}\right), 39.58\left(-\underline{\mathrm{CH}}_{2} \mathrm{~N}-\right)$, $33.45\left(-\underline{C H}_{2} \mathrm{~S}-\right), 32.04\left(-\underline{C H}_{2} \mathrm{CO}\right), 29.14\left(\underline{\mathrm{CH}}_{2}\right), 28,67$ $\left(\underline{\mathrm{CH}}_{2}\right), 25.44\left(\underline{\mathrm{CH}}_{3}\right)$; HRMS (EI-MS): $\mathrm{m} / z$ calculated for $\mathrm{C}_{19} \mathrm{H}_{25} \mathrm{BrN}_{6} \mathrm{O}_{6} \mathrm{~S}[\mathrm{M}+\mathrm{H}]^{+}$545.0811; found 545.0812; Green chemistry metrics: E-factor 1.874, ME 0.352.
$N^{2}$-[(2-(4-Methoxyphenyl)-4-oxo-1,3-thiazolidin-3-yl) propionyl]-nitro-L-arginine methyl ester (6f)

Light yellow cristals, mp $95{ }^{\circ} \mathrm{C}$, yield: $86 \%$; IR ( $\mathrm{Zn} / \mathrm{Se}$ crystal, $\left.\mathrm{cm}^{-1}\right)$ : $3298(-\mathrm{NH}) ; 3001,783\left(=\mathrm{C}-\mathrm{H}_{\text {phenyl }}\right) ; 1740$ $\left(\mathrm{COOCH}_{3}\right) ; 1651(-\mathrm{CONH}) ; 1628\left(\mathrm{C}=\mathrm{O}_{\text {thiazolidine-4-one }}\right)$; $1609\left(-\mathrm{C}=\mathrm{C}-{ }_{\text {phenyl }}\right) ; 1346,1111(-\mathrm{C}-\mathrm{N}-)$; 1246, 725 ($\left.\mathrm{CH}_{2}-\right) ; 1153\left(-\mathrm{OCH}_{3}\right) ; 687(\mathrm{C}-\mathrm{S}) ;{ }^{1} \mathrm{H}-\mathrm{NMR}(\delta \mathrm{ppm})$ :

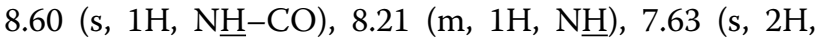
$\mathrm{NH}), 7.38-7.18(\mathrm{~m}, 2 \mathrm{H}, \mathrm{Ar}-\mathrm{H}), 6.91(\mathrm{~d}, \mathrm{~J}=8.6 \mathrm{~Hz}, 2 \mathrm{H}$, Ar-H), 5.73 (d, J = 42.3 Hz, 1H, -N-Cㅍ-S), 4.65-4.53 (m, $1 \mathrm{H}, \mathrm{CH}_{\underline{2}}-\mathrm{S}$ ), 3.90-3.84 (m, $1 \mathrm{H}, \mathrm{CH}_{2}-\mathrm{S}$ ), 3.82 (s, $3 \mathrm{H}$, $\mathrm{C}_{\underline{3}}$ ester), $3.76\left(\mathrm{~d}, \mathrm{~J}=3.3 \mathrm{~Hz}, 3 \mathrm{H}, \mathrm{OC}_{\underline{3}}\right), 3.71(\mathrm{~s}, 1 \mathrm{H}$, C프- $\mathrm{COOCH}_{3}$ ), 3.52-3.27 (m, 2H, $\mathrm{CH}_{2}$ arg), 3.22-3.01 $\left(\mathrm{m}, 1 \mathrm{H}, \mathrm{N}-\mathrm{CH}_{2}\right), 2.61-2.48\left(\mathrm{~m}, 1 \mathrm{H}, \mathrm{N}-\mathrm{CH}_{2}\right), 2.42-2.27$ (m, 1H, $\mathrm{CH}_{2}-\mathrm{CO}$ ), 1.95-1.85 (m, $1 \mathrm{H}, \mathrm{CH}_{\underline{2}}-\mathrm{CO}$ ), 1.77$1.54\left(\mathrm{~m}, 4 \mathrm{H}, 2 \underline{\mathrm{C}}_{2}\right.$ arg); ${ }^{13} \mathrm{C}-\mathrm{NMR}(\delta \mathrm{ppm}): 172.40$, 171.99, 160.63 (3C, $\underline{\mathrm{CO}}), 159.62\left(\mathrm{C}_{\text {guanid }}\right), 130.67,130.11$ $\left(2 \mathrm{C}, \mathrm{C}_{\mathrm{Ar}}\right), 128.96\left(2 \mathrm{C}, \underline{\mathrm{CH}}_{\mathrm{Ar}}\right), 114.70\left(2 \mathrm{C}, \underline{\mathrm{C}} \mathrm{H}_{\mathrm{Ar}}\right), 63.80$ $(\mathrm{S}-\underline{\mathrm{C}} \mathrm{H}-\mathrm{N}), 55.71(\underline{\mathrm{CH}}), 52.92\left(\mathrm{O}_{\mathrm{CH}}\right), 40.46\left(\underline{\mathrm{CH}}_{2}\right)$, $39.56\left(-\underline{C H}_{2} \mathrm{~N}-\right), 33.94\left(-\underline{C H}_{2} \mathrm{~S}-\right), 32.92\left(\underline{\mathrm{CH}}_{2}\right), 29.51$ (- $\left.\underline{\mathrm{CH}}_{2} \mathrm{CO}\right), 23.68\left(\underline{\mathrm{CH}}_{2}\right), 21.45\left(\underline{\mathrm{CH}}_{3}\right)$; HRMS (EI-MS): $m / z$ calculated for $\mathrm{C}_{20} \mathrm{H}_{28} \mathrm{~N}_{6} \mathrm{O}_{7} \mathrm{~S}[\mathrm{M}+\mathrm{H}]^{+}$497.1813; found 497.1813; Green chemistry metrics: E-factor 1.506, ME 0.403 .

\section{$N^{2}$-[(2-(3-Methoxyphenyl)-4-oxo-1,3-thiazolidin-3-yl) propionyl]-nitro-L-arginine methyl ester $(\mathbf{6 g})$}

Light pink cristals, mp $103{ }^{\circ} \mathrm{C}$, yield: $78 \%$; IR ( $\mathrm{Zn} /$ Se crystal, cm $\left.{ }^{-1}\right)$ : $3298(-\mathrm{NH})$; 3001, 771 (=C-H phenyl); 2951, 1254, $725\left(-\mathrm{CH}_{2}-\right)$; $1740\left(\mathrm{COOCH}_{3}\right) ; 1651$ $(-\mathrm{CONH}) ; 1647\left(\mathrm{C}=\mathrm{O}_{\text {thiazolidine-4-one }}\right) ; 1601 \quad\left(-\mathrm{C}=\mathrm{C}-{ }_{\text {phe- }}\right.$ nyl); 1338, 1041 (-C-N-); $1149\left(-\mathrm{OCH}_{3}\right) ; 694(\mathrm{C}-\mathrm{S}) ;{ }^{1} \mathrm{H}-$ NMR $(\delta \mathrm{ppm}): 8.52(\mathrm{~s}, 1 \mathrm{H}, \mathrm{N} \underline{\mathrm{H}}-\mathrm{CO}), 8.09(\mathrm{~m}, 1 \mathrm{H}, \mathrm{N} \underline{\mathrm{H}})$, $7.52-7.47(\mathrm{~m}, 2 \mathrm{H}, \mathrm{N} \underline{\mathrm{H}}), 7.32(\mathrm{t}, \mathrm{J}=7.9 \mathrm{~Hz}, 1 \mathrm{H}, \mathrm{Ar}-\mathrm{H})$, 6.97-6.85 (m, 2H, Ar-H), 6.74 (dd, J = 31.2, 7.7 Hz, $1 \mathrm{H}, \mathrm{Ar}-\mathrm{H}), 5.72(\mathrm{~d}, \mathrm{~J}=63.5,5.7 \mathrm{~Hz}, 1 \mathrm{H},-\mathrm{N}-\mathrm{C} \underline{\mathrm{H}}-\mathrm{S})$, 4.68-4.54 (m, 1H, $\left.\mathrm{CH}_{\underline{2}}-\mathrm{S}\right)$, 3.94-3.85 (m, $\left.1 \mathrm{H}, \mathrm{CH}_{\underline{2}}-\mathrm{S}\right)$, 3.82 (s, $3 \mathrm{H}, \mathrm{CH}_{3}$ ester), 3.79-3.78 (d, J = 3.5 Hz, $3 \mathrm{H}$, $\left.\mathrm{OC}_{\underline{H}}\right), 3.72\left(\mathrm{~s}, 1 \mathrm{H}, \mathrm{C} \underline{\mathrm{H}}-\mathrm{COOCH}_{3}\right), 3.57-3.29(\mathrm{~m}, 2 \mathrm{H}$, $\mathrm{C}_{2} \underline{\mathrm{H}}_{2} \mathrm{arg}$ ), 3.25-3.05 (m, 1H, N-CH $\mathrm{CH}_{2}$, 2.55 (dt, J = 7.6, $\left.6.9 \mathrm{~Hz}, 1 \mathrm{H}, \mathrm{N}-\mathrm{CH}_{2}\right), 2.45-2.32\left(\mathrm{~m}, 1 \mathrm{H}, \mathrm{CH}_{2}-\mathrm{CO}\right)$, $1.91\left(\mathrm{dd}, \mathrm{J}=8.5,4.0 \mathrm{~Hz}, 1 \mathrm{H}, \mathrm{CH}_{2}-\mathrm{CO}\right), 1.77-1.55$ $\left(\mathrm{m}, 4 \mathrm{H}, 2 \underline{\mathrm{CH}}_{2} \mathrm{arg}\right) ;{ }^{13} \mathrm{C}-\mathrm{NMR}(\delta \mathrm{ppm}): 172.37,170.65$, 160.39 (3C, $\underline{\mathrm{C}} \mathrm{O}), 159.66\left(\mathrm{C}_{\text {guanid }}\right), 140.76,130.52(2 \mathrm{C}$, $\left.\mathrm{C}_{\mathrm{Ar}}\right), 119.47,114.98,114.75,113.11\left(4 \mathrm{C}, \underline{\mathrm{CH}}_{\mathrm{Ar}}\right), 64.07$ $(\mathrm{S}-\underline{\mathrm{C}} \mathrm{H}-\mathrm{N}), 55.56(\underline{\mathrm{CH}}), 53.01\left(\mathrm{O}_{\mathrm{CH}}\right), 40.57\left(\underline{\mathrm{CH}}_{2}\right)$, $39.79\left(-\mathrm{CH}_{2} \mathrm{~N}-\right), 34.05\left(-\underline{\mathrm{CH}}_{2} \mathrm{~S}-\right), 31.94\left(-\underline{\mathrm{CH}}_{2} \mathrm{CO}\right)$, 29.65, $24.27\left(2 \underline{C H}_{2}\right), 21.34\left(\underline{C H}_{3}\right) ;$ HRMS (EI-MS): $m / z$ calculated for $\mathrm{C}_{20} \mathrm{H}_{28} \mathrm{~N}_{6} \mathrm{O}_{7} \mathrm{~S}[\mathrm{M}+\mathrm{H}]^{+}$497.1813; found 497.1812; Green chemistry metrics: E-factor 3.767, ME 0.213 . 
$N^{2}$-[(2-(2-Methoxyphenyl)-4-oxo-1,3-thiazolidin-3-yl) propionyl]-nitro-L-arginine methyl ester (6h)

Light yellow cristals, mp $115{ }^{\circ} \mathrm{C}$, yield: $76 \%$; IR (Zn/ Se crystal, cm $\left.{ }^{-1}\right)$ : $3298(-\mathrm{NH}) ; 3078,771(=\mathrm{C}-\mathrm{H}$ phenyl); 2947, 1242, $725\left(-\mathrm{CH}_{2}-\right) ; 1736\left(\mathrm{COOCH}_{3}\right) ; 1647$ $(-\mathrm{CONH}) ; 1628 \quad\left(\mathrm{C}=\mathrm{O}_{\text {thiazolidine-4-one }}\right) ; 1597 \quad(-\mathrm{C}=\mathrm{C}-$ phenyl); 1350, 1049 (-C-N-); $1153\left(-\mathrm{OCH}_{3}\right) ; 683(\mathrm{C}-\mathrm{S})$; ${ }^{1} \mathrm{H}-\mathrm{NMR}(\delta \mathrm{ppm}): 8.48(\mathrm{~s}, 1 \mathrm{H}, \mathrm{NH}-\mathrm{CO}), 7.94(\mathrm{~m}, 1 \mathrm{H}$, $\mathrm{N} \underline{\mathrm{H}}), 7.50(\mathrm{~s}, 2 \mathrm{H}, \mathrm{N} \underline{\mathrm{H}}), 7.34(\mathrm{t}, \mathrm{J}=7.9 \mathrm{~Hz}, 1 \mathrm{H}, \mathrm{Ar}-\mathrm{H})$, 7.15 (dd, J = 13.1, 4.3 Hz, 1H, Ar-H), 7.05-6.92 (m, 1H, Ar-H), 6.84-6.74 (m, 1H, Ar-H), 6.08 (d, J = 37.2 Hz, $1 \mathrm{H},-\mathrm{N}-\mathrm{C} \underline{\mathrm{H}}-\mathrm{S}), 4.69-4.51\left(\mathrm{~m}, 1 \mathrm{H}, \mathrm{CH}_{2}-\mathrm{S}\right), 3.94(\mathrm{t}$, $\left.\mathrm{J}=7.2 \mathrm{~Hz}, 1 \mathrm{H}, \mathrm{CH}_{2}-\mathrm{S}\right), 3.90-3.86$ (s, $3 \underline{\mathrm{H}}, \mathrm{C}_{\underline{3}}$ ester), $3.78\left(\mathrm{~d}, \mathrm{~J}=3.1 \mathrm{~Hz}, 3 \mathrm{H}, \mathrm{OCH}_{3}\right), 3.66-3.56(\mathrm{~m}, 1 \underline{\mathrm{H}}, \mathrm{CH}-$ $\mathrm{COOCH}_{3}$ ), 3.40 (dd, J = 69.6, $5.1 \mathrm{~Hz}, 2 \mathrm{H}, \mathrm{CH}_{2} \arg$ ), 3.11 (ddd, J = 11.9, 9.6, 6.4 Hz, $1 \mathrm{H}, \mathrm{N}-\mathrm{CH}_{2}$ ), 2.72-2.53 $\left(\mathrm{m}, 1 \mathrm{H}, \mathrm{N}-\mathrm{CH}_{2}\right), 2.46\left(\mathrm{dt}, \mathrm{J}=15.2,6.2 \mathrm{~Hz}, 1 \mathrm{H}, \mathrm{CH}_{2}-\right.$ $\mathrm{CO}), 2.01-1.86\left(\mathrm{~m}, 1 \mathrm{H}, \mathrm{CH}_{2}-\mathrm{CO}\right), 1.78-1.48(\mathrm{~m}, 4 \mathrm{H}$, $2 \underline{\mathrm{C}}_{2}$ arg); ${ }^{13} \mathrm{C}-\mathrm{NMR}(\delta \mathrm{ppm}): 172.98,171.33,164.37$ (3C, $\underline{\mathrm{CO}}), 159.94\left(\mathrm{C}_{\text {guanid }}\right), 157.47,130.82\left(2 \mathrm{C}, \mathrm{C}_{\mathrm{Ar}}\right)$, $127.38\left(\underline{\mathrm{CH}}_{\mathrm{Ar}}\right), 121.55\left(2 \mathrm{C}, \underline{\mathrm{CH}}_{\mathrm{Ar}}\right), 111.78\left(\underline{\mathrm{CH}}_{\mathrm{Ar}}\right), 63.95$ $(\mathrm{S}-\underline{\mathrm{C}} \mathrm{H}-\mathrm{N}), 56.21(\underline{\mathrm{CH}}), 53.30\left(\mathrm{O}_{\mathrm{CH}}\right), 40.81\left(\underline{\mathrm{CH}}_{2}\right)$, $39.19\left(-\underline{\mathrm{CH}}_{2} \mathrm{~N}-\right), 34.53\left(-\mathrm{CH}_{2} \mathrm{~S}-\right), 33.10\left(\underline{\mathrm{CH}}_{2}\right), 31.43$ (- $\left.\underline{\mathrm{CH}}_{2} \mathrm{CO}\right), 24.91\left(\underline{\mathrm{CH}}_{2}\right), 22.17\left(\underline{\mathrm{CH}}_{3}\right) ; \mathrm{RMS}$ (EI-MS): $m / z$ calculated for $\mathrm{C}_{20} \mathrm{H}_{28} \mathrm{~N}_{6} \mathrm{O}_{7} \mathrm{~S}[\mathrm{M}+\mathrm{H}]^{+}$497.1813; found 497.1814; Green chemistry metrics: E-factor 2.079, ME 0.325.

$N^{2}$-[(2-(3-Nitrophenyl)-4-oxo-1,3-thiazolidin-3-yl) propionyl]-nitro-L-arginine methyl ester (6i)

Light yellow cristals, mp $100{ }^{\circ} \mathrm{C}$, yield: $50 \%$; IR $(\mathrm{Zn} /$ Se crystal, $\left.\mathrm{cm}^{-1}\right)$ : $3302(-\mathrm{NH}) ; 3090,783$ (=C-H phenyl); 2951, 1257, $729\left(-\mathrm{CH}_{2}-\right) ; 1736\left(\mathrm{COOCH}_{3}\right) ; 1651$ $(-\mathrm{CONH}) ; 1632\left(\mathrm{C}=\mathrm{O}_{\text {thiazolidine-4-one }}\right) ; 1601 \quad(-\mathrm{C}=\mathrm{C}-$ phenyl); 1528, $1350\left(\mathrm{NO}_{2}\right) ; 1219,1095$ (-C-N-); 683 $(\mathrm{C}-\mathrm{S}) ;{ }^{1} \mathrm{H}-\mathrm{NMR}(\delta \mathrm{ppm}): 8.58(\mathrm{~s}, 1 \mathrm{H}, \mathrm{N} \underline{\mathrm{H}}-\mathrm{CO}), 8.21(\mathrm{~d}$, $\mathrm{J}=4.3 \mathrm{~Hz}, 2 \mathrm{H}, \mathrm{Ar}-\mathrm{H}), 8.03(\mathrm{~m}, 1 \mathrm{H}, \mathrm{N} \underline{\mathrm{H}}), 7.77-7.67(\mathrm{~m}$, $1 \mathrm{H}, \mathrm{Ar}-\mathrm{H}), 7.61(\mathrm{t}, \mathrm{J}=8.0 \mathrm{~Hz}, 1 \mathrm{H}, \mathrm{Ar}-\mathrm{H}), 7.49-7.34$

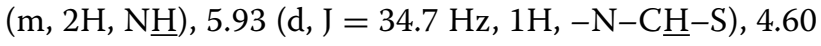
(s, $\left.1 \mathrm{H}, \mathrm{CH}_{2}-\mathrm{S}\right), 3.98-3.83\left(\mathrm{~m}, 1 \mathrm{H}, \mathrm{CH}_{2}-\mathrm{S}\right), 3.79$ (s, $3 \mathrm{H}$, $\mathrm{CH}_{3}$ ester), $3.76\left(\mathrm{~d}, \mathrm{~J}=4.6 \mathrm{~Hz}, 1 \mathrm{H}, \mathrm{C} \underline{\mathrm{H}}-\mathrm{COOCH}_{3}\right)$, 3.59-3.39 (m, 2H, $\underline{\mathrm{C}}_{2}$ arg), 3.36-3.22 (m, 1H, N$\left.\mathrm{CH}_{2}\right), 3.24-3.00\left(\mathrm{~m}, 1 \mathrm{H}, \mathrm{N}-\mathrm{CH}_{2}\right), 2.74-2.35(\mathrm{~m}, 2 \mathrm{H}$,

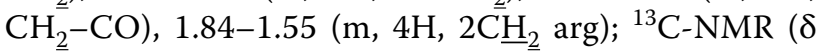
ppm): 173.24, 171.67, 168.34 (3C, $\underline{\mathrm{CO}}), 160.14\left(\mathrm{C}_{\text {guanid }}\right)$, 149.41, 142.55 (2C, $\left.\mathrm{C}_{\mathrm{Ar}}\right), 135.30,133.92,131.10,129.30$ $\left(4 \mathrm{C}, \underline{\mathrm{CH}}_{\mathrm{Ar}}\right), 63.58(\mathrm{~S}-\underline{\mathrm{CH}}-\mathrm{N}), 53.54(\underline{\mathrm{CH}}), 41.32\left(\underline{\mathrm{CH}}_{2}\right)$, $39.41\left(\underline{\mathrm{CH}}_{2} \mathrm{~N}-\right), 34.37\left(-\underline{\mathrm{CH}}_{2} \mathrm{~S}-\right), 33.48\left(\underline{\mathrm{CH}}_{2}\right), 31.94$ (- $\left.\underline{\mathrm{CH}}_{2} \mathrm{CO}\right), 24.27\left(\underline{\mathrm{CH}}_{2}\right), 22.37\left(\underline{\mathrm{CH}}_{3}\right)$; HRMS (EI-MS): $m / z$ calculated for $\mathrm{C}_{19} \mathrm{H}_{25} \mathrm{~N}_{7} \mathrm{O}_{8} \mathrm{~S}[\mathrm{M}+\mathrm{H}]^{+}$512.1558; found 512.1554; Green chemistry metrics: E-factor 3.687, ME 0.2134.
$N^{2}$-[(2-(2-Nitrophenyl)-4-oxo-1,3-thiazolidin-3-yl) propionyl]-nitro-L-arginine methyl ester (6j)

Yellow cristals, mp $95{ }^{\circ} \mathrm{C}$, yield: $98 \%$; IR (Zn/Se crystal, $\left.\mathrm{cm}^{-1}\right)$ : 3302 (-NH); 2983, 767 (=C-H $\left.\mathrm{H}_{\text {phenyl }}\right)$; 2954, 1257, $725\left(-\mathrm{CH}_{2}-\right) ; 1736\left(\mathrm{COOCH}_{3}\right) ; 1659(-\mathrm{CONH}) ; 1628$ $\left(\mathrm{C}=\mathrm{O}_{\text {thiazolidine-4-one }}\right) ; 1606 \quad\left(-\mathrm{C}=\mathrm{C}-{ }_{\text {phenyl }}\right) ; 1524,1342$ $\left(\mathrm{NO}_{2}\right) ; 1215,1115(-\mathrm{C}-\mathrm{N}-)$; $687(\mathrm{C}-\mathrm{S}) ;{ }^{1} \mathrm{H}-\mathrm{NMR}(\delta$

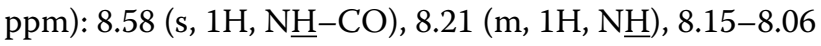
$(\mathrm{m}, 1 \mathrm{H}, \mathrm{Ar}-\mathrm{H}), 7.72$ (dd, J = 11.5, $3.8 \mathrm{~Hz}, 1 \mathrm{H}, \mathrm{Ar}-\mathrm{H})$, 7.52 (dd, J = 11.4, 4.0 Hz, 1H, Ar-H), 7.35-7.28 (m, 1H, Ar-H), 7.20-7.14 (m, 2H, NH) 6.30 (d, J = 23.8 Hz, $1 \mathrm{H}$, $-\mathrm{N}-\mathrm{C} \underline{\mathrm{H}}-\mathrm{S}), 4.57$ (d, J = 7.2 Hz, $\left.1 \mathrm{H}, \mathrm{CH}_{\underline{2}}-\mathrm{S}\right), 4.07-3.94$ (m, $1 \mathrm{H}, \mathrm{CH}_{2}-\mathrm{S}$ ), 3.77 (d, J = $13.6 \mathrm{~Hz}, 3 \mathrm{H}, \mathrm{C}_{\underline{3}}$ ester), $3.71-3.66\left(\mathrm{~m}, 1 \mathrm{H}, \mathrm{CH}-\mathrm{COOCH}_{3}\right), 3.61(\mathrm{dd}, \mathrm{J}=15.7$, $2.8 \mathrm{~Hz}, 2 \mathrm{H}, \mathrm{CH}_{2}$ arg), 3.49-3.28 (m, $\left.1 \mathrm{H}, \mathrm{N}-\mathrm{CH}_{2}\right), 3.17-$ $3.02\left(\mathrm{~m}, 1 \mathrm{H}, \underline{\mathrm{N}}-\mathrm{CH}_{2}\right), 2.71-2.47\left(\mathrm{~m}, 1 \mathrm{H}, \mathrm{CH}_{\underline{2}}-\mathrm{CO}\right)$, 2.02-1.85 (m, $\left.1 \mathrm{H}, \mathrm{CH}_{2}-\mathrm{CO}\right), 1.77-1.61\left(\mathrm{~m}, 4 \mathrm{H}, 2 \underline{\mathrm{CH}}_{2}\right.$ arg); ${ }^{13} \mathrm{C}-\mathrm{NMR}(\delta \mathrm{ppm}): 172.01,170.56,162.34(3 \mathrm{C}, \underline{\mathrm{CO}})$, 159.04 $\left(\mathrm{C}_{\text {guanid }}\right), 146.93,136.09\left(2 \mathrm{C}, \mathrm{C}_{\mathrm{Ar}}\right), 134.38,129.13$, 125.70, 116.24 (4C, $\left.\underline{\mathrm{CH}}_{\mathrm{Ar}}\right), 63.95(\mathrm{~S}-\underline{\mathrm{C}} \mathrm{H}-\mathrm{N}), 58.72$ $(\underline{\mathrm{CH}}), 40.21\left(\underline{\mathrm{CH}}_{2}\right), 39.33\left(-\underline{\mathrm{CH}}_{2} \mathrm{~N}-\right), 33.64\left(-\underline{\mathrm{CH}}_{2} \mathrm{~S}-\right)$, $31.27\left(\underline{\mathrm{CH}}_{2}\right), \quad 31.94 \quad\left(-\underline{\mathrm{CH}}_{2} \mathrm{CO}\right), 29.55\left(\underline{\mathrm{CH}}_{2}\right), 24.26$ $\left(\mathrm{CH}_{3}\right)$; HRMS (EI-MS): $m / z$ calculated for $\mathrm{C}_{19} \mathrm{H}_{25} \mathrm{~N}_{7} \mathrm{O}_{8} \mathrm{~S}$ $[\mathrm{M}+\mathrm{H}]^{+}$512.1558; found 512.1559; Green chemistry metrics: E-factor 1.218, ME 0.452.

\section{Biological evaluation \\ Antioxidant activity \\ DPPH radical scavenging assay}

The radical scavenging activity of the tested compounds towards 1,1-diphenyl-2-picrylhydrazyl (DPPH) radical was measured as described in literature [32] with minor modifications. The samples were dissolved in DMSO in order to form the stock solutions with the concentration of $20 \mathrm{mg} / \mathrm{mL}$. From the stock solutions there were taken different volumes $(50,100,150,200 \mu \mathrm{L})$ and completed up to $200 \mu \mathrm{L}$ with methanol, then it was added $2800 \mu \mathrm{L}$ of $0.1 \mathrm{mM}$ DPPH methanol solution. The resulting mixture was kept in the dark for 60 min after which the absorbance was read at $517 \mathrm{~nm}$ against methanol, used as a blank solution. The final concentration of sample in the test tube was $0.33,0.66,0.99$ and $1.32 \mathrm{mg} / \mathrm{mL}$ respectively. The DPPH radical-inhibiting capacity (radical scavenging ability) was calculated using the following formula:

Inhibition (scavenging activity) $\%=\left[\left(\mathrm{A}_{\mathrm{C}}-\mathrm{A}_{\mathrm{S}}\right) / \mathrm{A}_{\mathrm{C}}\right] \times 100$

where $\mathrm{A}_{\mathrm{C}}=$ absorbance of the DPPH solution, $\mathrm{A}_{\mathrm{S}}=$ absorbance of the sample. Vitamin $\mathrm{E}$ ( $\alpha$-tocopherol) was used as positive control and as references were used $\mathrm{NO}_{2}$-Arg-OMe and L-arginine, all three being processed 
in a similar manner with the samples. All determinations were performed in triplicate.

\section{ABTS radical scavenging assay}

The generation of radical cation $\mathrm{ABTS}^{+}$was carried out by treating the aqueous solution of $2,2^{\prime}$-azino-bis (3-ethylbenzothiazoline-6-sulfonic acid) (7 mM) with ammonium persulfate $(2.45 \mathrm{mM})$. The resulting mixture was kept in the dark for $16 \mathrm{~h}$ to promote the formation of $\mathrm{ABTS}^{+}$, as described in $[33,34]$. The $\mathrm{ABTS}^{+}$radical cation solution was diluted with ethanol to obtain an absorbance value of $0.7 \pm 0.02$ at $734 \mathrm{~nm}$. Different sample volumes $(10,15,25,50 \mu \mathrm{L})$ from a stock solution of $20 \mathrm{mg} / \mathrm{mL}$ in DMSO were mixed with DMSO to $50 \mu \mathrm{L}$ and then $1950 \mu \mathrm{L}$ of ABTS $^{+}$solution were added. The final concentration of sample in the test tube was 0.1 , $0.15,0.25$ and $0.50 \mathrm{mg} / \mathrm{mL}$ respectively. After 6 min the absorbance was measured at $734 \mathrm{~nm}$ against a blank (ethanol) and the radical scavenging capacity was calculated according to the following equation:

$$
\text { Scavenging activity } \%=\left[\left(\mathrm{A}_{\mathrm{C}}-\mathrm{A}_{\mathrm{S}}\right) / \mathrm{A}_{\mathrm{C}}\right] \times 100
$$

where $\mathrm{A}_{\mathrm{C}}=$ absorbance of $\mathrm{ABTS}^{+}$alcoholic solution; $A_{S}=$ absorbance of the samples, read at $6 \mathrm{~min}$ after the addition of the $\mathrm{ABTS}^{+}$solution. Vitamin $\mathrm{E}$ $(\alpha$-tocopherol) was used as positive control and as references were used $\mathrm{NO}_{2}$-Arg-OMe and L-arginine, all three being processed in a similar manner with the samples. All determinations were performed in triplicate.

\section{Phosphomolydenum reducing antioxidant power (PRAP) assay}

The total antioxidant activity of tested compounds was evaluated using the phosphomolybdenum method according to the procedure described in the literature [35] with minor modifications. For each compound was prepared a stock solution with the concentration of $20 \mathrm{mg} / \mathrm{mL}$ in DMSO, from which there were used different volumes $(20,40,60,80 \mu \mathrm{L})$ and completed with DMSO up to $200 \mu \mathrm{L}$. Over these samples it was added $2 \mathrm{~mL}$ of the reagent solution $(0.6 \mathrm{M}$ sulfuric acid, $28 \mathrm{mM}$ disodium hydrogen phosphate, and $4 \mathrm{mM}$ ammonium molybdate). The samples were incubated at $95{ }^{\circ} \mathrm{C}$ for $90 \mathrm{~min}$ at drying stove (oven). The final concentration of sample in the test tube was $0.18,0.36,0.54$ and $0.72 \mathrm{mg} /$ $\mathrm{mL}$ respectively. After cooling to room temperature, the absorbance was read at $695 \mathrm{~nm}$ against a blank $(200 \mathrm{~mL}$ DMSO $+2 \mathrm{~mL}$ reagent). Vitamin $\mathrm{E}$ ( $\alpha$-tocopherol) was used as positive control and as references were used $\mathrm{NO}_{2}$-Arg-OMe and L-arginine, all three being processed in a similar manner with the samples. All determinations were performed in triplicate.

\section{Ferric reducing antioxidant power (FRAP) assay}

The ferric reducing antioxidant power of the compounds was quantified by the method described by [36] with slight modifications. The compounds were tested at different concentrations $(20,10,5,2.5 \mathrm{mg} / \mathrm{mL})$. To $0.5 \mathrm{~mL}$ of samples of each concentration it was added $0.5 \mathrm{~mL}$ of $0.2 \mathrm{M}$ phosphate buffer $\mathrm{pH}$ 6.6. The reaction was then initiated by the addition of $0.5 \mathrm{~mL}$ of potassium ferricyanide $1 \% \mathrm{w} / \mathrm{v}$, after which the samples are incubated at $50{ }^{\circ} \mathrm{C}$ (oven) for $20 \mathrm{~min}$ and the completion of the reaction takes place by addition of $0.5 \mathrm{~mL}$ trichloroacetic acid $10 \% \mathrm{w} / \mathrm{v} .1 \mathrm{~mL}$ from the resulting solution of each sample was diluted with $1 \mathrm{~mL}$ double distilled deionised water and finally $0.2 \mathrm{~mL}$ of ferric chloride $0.1 \% \mathrm{w} / \mathrm{v}$ was added. The final concentration of sample in the test tube was $4.5454,2.2727,1.1360,0.5681 \mathrm{mg} / \mathrm{mL}$ respectively. The mixture was left at room temperature for $10 \mathrm{~min}$ and then the absorbance was measured at $700 \mathrm{~nm}$ against a blank solution prepared similar to the sample, which contain $0.5 \mathrm{~mL}$ DMSO instead $0.5 \mathrm{~mL}$ sample. Vitamin E $(\alpha$-tocopherol) was used as positive control and as references were used $\mathrm{NO}_{2}$-Arg-OMe and L-arginine, all three being processed in a similar manner with the samples. All determinations were performed in triplicate.

\section{Antibacterial/antifungal assays}

Agar disc diffusion method Antibacterial and antifungal activity of the $\mathbf{6} \mathbf{a}-\mathbf{j}$ derivatives expressed as diameter of inhibition area was evaluated by the standard disk diffusion assay according to described protocols [42]. Prior to use, the strains (bacteria and yeasts) were diluted in sterile $0.9 \% \mathrm{NaCl}$ until the turbidity was equivalent to McFarland standard no. 0.5 (106 CFU/mL). The suspensions were further diluted 1:10 in Mueller-Hinton agar for bacteria and Sabouraud agar for fungi and then spread on sterile Petri plates ( $25 \mathrm{~mL} /$ Petri plate). Sterile stainless steel cylinders (5 mm internal diameter; $10 \mathrm{~mm}$ height) were applied on the agar surface in Petri plates. In each cylinder $200 \mu \mathrm{L}$ of sample solutions in DMSO $(20 \mathrm{mg} / \mathrm{mL})$ was added. As positive control there were used commercial available discs containing ampicillin (25 mcg/disc), chloramphenicol (30 mcg/disc) and nystatin $(100 \mathrm{mcg} /$ disc). DMSO was used as a negative control. The plates were incubated at $37^{\circ} \mathrm{C}$ for $24 \mathrm{~h}$ (bacteria) and at $24{ }^{\circ} \mathrm{C}$ for $48 \mathrm{~h}$ (fungi). The diameters of inhibition area developed after the incubation were measured.

The broth micro-dilution method The minimum inhibitory concentration (MIC) and the minimum bactericidal/fungicidal concentration (MBC/MFC) against bacteria and fungi respectively were determined by the two-fold dilution method, with minor modification [38]. 
The active cultures of the bacteria and fungi were prepared by transferring the loopful of cells from the stock culture to the conical flasks containing Mueller-Hinton broth for bacteria or Sabouraud broth for fungi. The cultures were incubated at $37^{\circ} \mathrm{C}$ for $24 \mathrm{~h}$ (bacteria) and at $24{ }^{\circ} \mathrm{C}$ for $48 \mathrm{~h}$ (fungi) and then were diluted with fresh media to obtain an optical density value of $106 \mathrm{CFU} /$ $\mathrm{mL}$. Different dilutions of the $6 \mathrm{a}-\mathrm{j}$ derivatives made in the Mueller-Hinton broth (bacteria) and in Sabourand broth (fungi) were prepared in a 96-well microplate by the twofold dilution method in the concentration range of $10,5,2.5,1.25,0.625,0.312,0.156,0.078,0.039$, $0.0195,0.009$ and $0.0048 \mathrm{mg} / \mathrm{mL}$. Then $10 \mu \mathrm{L}$ of each strain $(106 \mathrm{CFU} / \mathrm{mL})$ was inoculated onto the microplates. The plates were incubated again at $37^{\circ} \mathrm{C}$ for $24 \mathrm{~h}$. The lowest concentrations of the tested compounds which did not show any visual growth of the test strain, were determined as the MICs, which were expressed in $\mathrm{mg} / \mathrm{mL}$. For the determination of MBCs and MFCs, the MIC and the next higher concentrations of the sample were selected, spread on the agar plates, and incubated at $37^{\circ} \mathrm{C}$ for $24 \mathrm{~h}$. The concentration of the tested compounds, which did not show any growth of the microorganism on the agar plates, was determined as the $\mathrm{MBC} /$ MFC and expressed in $\mathrm{mg} / \mathrm{mL}$. Each determination was performed in triplicate.

\section{Conclusions}

The present work is centered on the synthesis and biological evaluation of new thiazolidine-4-ones derived from the methyl ester of nitro-L-arginine. The structure of the compounds was proven using spectral methods (IR, ${ }^{1} \mathrm{H}$ NMR, ${ }^{13} \mathrm{C}$-NMR, MS). The antioxidant activity was quantified using four in vitro tests: DPPH/ABTS scavenging assays and ferric/phosphomolybdenum reducing antioxidant power assays. The methoxy-substituted derivatives, 6h $\left(\mathrm{R}=2-\mathrm{OCH}_{3}\right)$ and $\mathbf{6 g}\left(3-\mathrm{OCH}_{3}\right)$, showed a high free radical scavenging ability, both for DPPH and ABTS radicals. A good influence was exerted also by the nitro and bromo substitution. The 2-nitro-derivative, $\mathbf{6 j}$, showed the best ABTS scavenging ability while the 4-bromoderivative, 6e, presented the best ferric and phosphomolybdenum reducing antioxidant power. The compound $\mathbf{6 j}$ also showed a good antibacterial and antifungal activity. It was the most active on $S$. aureus, $S$. lutea and $P$. aeruginosa and Candida spp. respectively. The encouraging preliminary results support the antioxidant and antibacterial/antifungal potential of the synthesized compounds and their possible applications in several diseases mediated by reactive oxygen species (ROS) and susceptible to infections such as wound healing from burns.

\section{Authors' contributions}

A-TP, FB, SR and LP designed research; A-TP, MA, IMV, MD and SC performed research; A-TP, FB, SR and LP analyzed the spectral data; A-TP, CT and LP analyzed the biological data; A-TP, LP, FB and SR wrote the paper. All authors read and approved the final manuscript.

\section{Author details}

${ }^{1}$ Department of Pharmaceutical Chemistry, Faculty of Pharmacy, University of Medicine and Pharmacy "Grigore T. Popa", 16 University Street, 700115 lasi, Romania. ${ }^{2}$ Institut de Chimie Organique et Analytique - ICOA UMR7311, Pôle de chimie, Rue de Chartres, 45100 Orléans, France. ${ }^{3}$ Department of Microbiology, Faculty of Pharmacy, University of Medicine and Pharmacy "Grigore T. Popa", 16 University Street, 700115 lasi, Romania.

\section{Acknowledgements}

The research was funded by POSDRU Grant No. 159/1.5/S/136893 grant with title "Parteneriat strategic pentru creşterea calităţii cercetării ştiinţifice din universităţile medicale prin acordarea de burse doctorale şi postdoctorale-DocMed.Net_2.0" (partially) and by a Grant of the Romanian National Authority for Scientific Research, CNCS_UEFISCDI, Project Number PNII-ID-PCE-2011-3-0906.

\section{Competing interests}

The authors declare that they have no competing interests.

Received: 11 November 2015 Accepted: 21 January 2016 Published online: 04 February 2016

\section{References}

1. Gad MZ (2010) Anti-aging effects of L-arginine (review). J Adv Res 1:169-177

2. Wu G, Jaeger LA, Bazer FW, Rhoads JM (2004) Arginine deficiency in preterm infants: biochemical mechanisms and nutritional implications. J Nutr Biochem 15:332-451

3. Gogoi M, Datey A, Wilson K, Chakravortty D (2016) Dual role of arginine metabolism in establishing pathogenesis. Curr Opin Microbiol 2:43-48

4. Kirk SJ, Hurson M, Regan MC, Holt DR, Wasserkrug HL, Barbul A (1993) Arginine stimulates wound healing and immune function in elderly human beings. Surgery 114:155-159

5. Nigris F, Lerman LO, Ignarro SW, Sica G, Lerman A, Palinski W, Ignarro LJ, Napoli C (2003) Beneficial effects of antioxidants and L-arginine on oxidation-sensitive gene expression and endothelial NO synthase activity at sites of disturbed shear stress. Proc Natl Acad Sci USA 100:1420-1425

6. Rocha J, Peixoto M, Jancar S, Cunha F, Ribeiro R, Rocha F (2002) Dual effect of nitric oxide in articular inflammatory pain in zymosan-induced arthritis in rats. Br J Pharmacol 136:588-596

7. Klamer D, Palsson E, Wass C, Archer T, Engel JA, Svensson L (2005) Antagonism of the nitric oxide synthase inhibitor, L-NAME, of the effects of phencyclidine on latent inhibition in taste aversion conditioning. Behav Brain Res 161(1):60-68

8. Rawls SM, Rodriguez T, Baron DA, Raffa RB (2006) A nitric oxide synthase inhibitor (L-NAME) attenuates abstinence-induced withdrawal from both cocaine and a cannabinoid agonist (WIN 55212-2) in Planaria. Brain Res $1: 82-87$

9. Wallner S, Hermetter A, Mayer B, Wascher TC (2001) The alpha-amino group of L-arginine mediates its antioxidant effects. Eur J Clin Invest 31:98-102

10. Tripathi P, Misra MK (2009) Therapeutic role of L-arginine on free radical scavenging system in ischemic heart diseases. Indian J Biochem Biophys 46:498-502

11. Kim JO, Noh J-K, Thapa RK, Hasan N, Choi M, Kim JH, Lee J-H, Ku SK, Yoo J-W (2015) Nitric oxide-releasing chitosan film for enhanced antibacterial and in vivo wound-healing efficacy. Int J Biol Macromol 79:217-225

12. Debats IBJG, Wolfs TGAM, Gotoh T, Cleutjens JPM, Peutz-Kootstra CJ, Van der Hulst R (2009) Role of arginine in superficial wound healing. Nitric Oxide 15:147-156 
13. Jing Y, Horky L, Friedlich AL, Ying S, Rogers JT, Xudong H (2009) L-Arginine and Alzheimer's disease. Int J Clin Exp Pathol 2:211-238

14. Pekarova M, Lojek A (2015) The crucial role of L-arginine in macrophage activation: what you need to know about it. Life Sci 137:44-48

15. Witte MB, Barbul A (2003) Arginine physiology and its implications for wound healing. Wound Repair Regen 11:419-423

16. Evans RW, Fernstrom JD, Thompson J, Morris SM, Kuller LH (2004) Biochemical responses of healthy subjects during dietary supplementation with L-arginine. J Nutr Biochem 15:534-539

17. Das UN (2015) Nutritional factors in the prevention and management of coronary artery disease and heart failure. Nutrition 31(2):283-291

18. Panza JA, Casino PR, Badar DM, Quyyumi AA (1993) Effect of increased availability of endothelium-derived nitric oxide precursor on endothelium-dependent vascular relaxation in normal subjects and in patients with essential hypertension. Circulation 87:1475-1481

19. Tripathi AC, Gupta SJ, Fatima GN, Sonar PK, Verma A, Saraf SK (2014) 4-Thiazolidinones: the advances continue. Eur J Med Chem 72:52-77

20. Isloor A, Sunil D, Shetty P, Malladi S, Pai K, MaliyakkI N (2013) Synthesis, characterization, anticancer, antioxidant activity of some new thiazolidin4-ones in MCF-7 cells. Med Chem Res 22:758-767

21. Šarkanj B, Molnar M, Ćačić M, Gille L (2013) 4-Methyl-7-hydroxycoumarin antifungal and antioxidant activity enhancement by substitution with thiosemicarbazide and thiazolidinone moieties. Food Chem 139:488-495

22. Mei-Hsiu S, Fang-Ying K (2004) Syntheses and evaluation of antioxidant activity of sydnonyl substituted thiazolidinone and thiazoline derivatives. Bioorg Med Chem 12:4633-4643

23. Apostolidis I, Liaras K, Geronikaki A, Hadjipavlou-Litina D, Gavalas A, Soković M (2013) Synthesis and biological evaluation of some 5-arylidene-2-(1,3-thiazol-2-yl-imino)-1,3-thiazolidin-4-ones as dual antiinflammatory/antimicrobial agents. Bioorg Med Chem 21:532-539

24. Ramachandran R, Rani M, Kabilan S (2009) Design, synthesis and biological evaluation of novel 2-[(2,4-diaryl-3-azabicyclo[3.3.1]nonan-9-ylidene) hydrazono]-1,3-thiazolidin-4-ones as a new class of antimicrobial agents. Bioorg Med Chem Lett 19:2819-2823

25. Liesen AP, de Aquino TM, Carvalho CS, Lima VT, de Araújo JM, de Lima JG, de Faria AR, de Melo EJ, Alves AJ, Alves EW, Alves AQ, Góes AJ (2010) Synthesis and evaluation of anti-toxoplasma gondii and antimicrobial activities of thiosemicarbazides, 4-thiazolidinones and 1,3,4-thiadiazoles. Eur J Med Chem 45:3685-3689

26. Aquino TM, Liesen AP, Da Silva RE, Lima VT, Carvalho CS, Faria AR, Araujo M, Lima JG, Alves AJ, Melo EJ, Goes AJ (2008) Synthesis, anti-Toxoplasma gondii and antimicrobial activities of benzaldehyde 4-phenyl-3-thiosemicarbazones and 2-[(phenylmethylene) hydrazono]-4-oxo-3-phenyl5-thiazolidine acetic acids. Bioorg Med Chem 16:446-456

27. Revelant G, Huber-Villaume S, Dunand S, Kirsch G, Schohn H, Hesse S (2015) Synthesis and biological evaluation of novel 2-heteroarylimino1,3-thiazolidin-4-ones as potential anti-tumor agents. Eur J Med Chem 94:102-112

28. Ottanà R, Maccari R, Giglio M, Del Corso A, Cappiello M, Mura U, Cosconati S, Marinelli L, Novellino E, Sartini S, La Motta C, Da Settimo F (2011) Identification of 5-arylidene-4-thiazolidinone derivatives endowed with dual activity as aldose reductase inhibitors and antioxidant agents for the treatment of diabetic complications. Eur J Med Chem 46:2797-2806

29. Khanna V, Kanji S, Nampurath GK, Chamallamudi MR, Mathew SP, Zachariah RT (2008) Assessment of hypolipidaemic activity of three thiazolidin4-ones in mice given high-fat diet and fructose. Chem Biol Interact 171:363-368

30. Verma RP, Hansch C (2007) Matrix metalloproteinases (MMPs): chemicalbiological functions and (Q)SARs. Bioo. Med Chem 15:2223-2268

31. Apotrosoaei M, Vasincu I, Drăgan O, Buron F, Routier S, Profire L (2014) Design, synthesis and the biological evaluation of 1,3-thiazolidine-4ones based on 4-amino-2,3-dimethyl-1-phenyl-3-pyrazolin-5one scaffold. Molecules 19:13824-13847

32. Schaich KM, Tian X, Xie J (2015) Hurdles and pitfalls in measuring antioxidant efficacy: a critical evaluation of ABTS, DPPH, and ORAC assays. J Funct Foods 14:111-125

33. Sadiq A, Mahmood F, Ullah F, Ayaz M, Ahmad S, Haq FU, Khan G, Jan MS (2015) Synthesis, anticholinesterase and antioxidant potentials of ketoesters derivatives of succinimides: a possible role in the management of Alzheimer's. Chem Cent J 9:31

34. Tabassum S, Kumara THS, Jasinski JP, Millikan SP, Yathirajan HS, Ganapathy PSS, Sowmya HBV, More SS, Nagendrappa G, Kaur M, Jose L (2014) Synthesis, crystal structure, ABTS radical-scavenging activity, antimicrobia and docking studies of some novel quinoline derivatives. J Mol Struct 1070:10-20

35. Cacic M, Molnar M, Sarkanj B, Has-Schon E, Rajkovic V (2010) Synthesis and antioxidant activity of some new coumarinyl-1,3-thiazolidine-4-ones. Molecules 15:6795-6809

36. Lungu Apetrei C, Tuchilus C, Aprotosoaie AC (2011) Chemical, antioxidant and antimicrobial investigations of Pinus cembra L. bark and needles. Molecules 16:7773-7788

37. Kubo I, Fujita K, Kubo A, Nihei K, Ogura T (2004) Antibacterial activity of coriander volatile compounds against Salmonella choleraesuis. J Agric Food Chem 52:3329-3332

38. Clinical and Laboratory Standards Institute(2015) Performance standards for antimicrobial susceptibility testing; twenty-fifth informational supplement. M100 S25. Clinical and laboratory standards institute, Wayne, PA

39. Andrews JM (2001) Determination of minimum inhibitory concentrations. J Antimicrob Chemother 48(S1):5-16

40. Protti S, Dondi D, Fagnoni M, Albini A (2009) Assessing photochemistry as a green synthetic method. Carbon-carbon bond forming reactions. Green Chem 11:239-249

41. Sambarkar PB, Patil AC (2012) Synthesis of amides from acid and amine using coupling reagents. J Curr Pharm Res 10:22-24

42. Brown DFJ, Blowers R (1978) Disc methods of sensitivity testing and other semiquantitative methods. In: Reeves DS, Phillips I, Williams JD, Wise $\mathrm{R}$ (eds) Laboratory methods in antimicrobial chemotherapy. Churchill Livingstone Edinburgh, UK, pp 8-23

\section{Submit your manuscript to a SpringerOpen ${ }^{\circ}$ journal and benefit from:}

- Convenient online submission

- Rigorous peer review

- Immediate publication on acceptance

- Open access: articles freely available online

- High visibility within the field

- Retaining the copyright to your article

Submit your next manuscript at $>$ springeropen.com 